\title{
Stable discretization of scalar and constrained vectorial Perona-Malik equation
}

\author{
SÖREN BARTELS ${ }^{\dagger}$ \\ Institut für Numerische Simulation, Universität Bonn, \\ Wegelerstr. 6, D-53115 Bonn, Germany \\ ANDREAS PROHL \\ Mathematisches Institut, Universität Tübingen, \\ Auf der Morgenstelle 10, D-72076 Tübingen, Germany
}

[Received 10 April 2006 and in revised form 8 August 2007]

\begin{abstract}
We survey recent results on analysis and numerics of the scalar Perona-Malik equation. A vectorial Perona-Malik equation is introduced to evolve unit vector fields for directional diffusion. For both cases, scalar and vectorial, fully discrete schemes are proposed which fulfill a discrete energy law, and satisfy a discrete sphere constraint in the vectorial case. Computational experiments are provided to illustrate quantitative behaviors, and compare with scalar total variation flow and heat flow of $p$ harmonic maps.
\end{abstract}

2000 Mathematics Subject Classification: 65M12, 65M60, 35K55, 35Q35.

Keywords: Total variation; Perona-Malik; $p$-harmonic map; finite elements, full discretization; discrete energy law.

\section{Introduction}

Regularization of multivalued images with PDE's is an active research area, which includes denoising/edge enhancement, segmentation, and inpainting of digital color images, and restoration of optical flow and direction fields, or fields of diffusion tensors in magnetic resonance imaging, for example. The goal in image segmentation and edge detection is to decompose a given image into regions that are essentially homogeneous (with little variation in color or brightness); these regions should be separated by sharp boundaries (edges). In this paper, we study directional diffusion, where a unit vector field $\mathbf{u}_{0}: \Omega \rightarrow \mathbb{S}^{2}$ is anisotropically diffused by a vectorial Perona-Malik equation into the sphere $\mathbb{S}^{2}$; this program is motivated by recent models, which suggest independent processing of brightness $\left|\mathbf{u}_{0}\right|: \Omega \rightarrow \mathbb{R}$ and chromaticity $\mathbf{u}_{0} /\left|\mathbf{u}_{0}\right|: \Omega \rightarrow \mathbb{S}^{2}$ of colored RGBimages $\mathbf{u}_{0}: \Omega \rightarrow \mathbb{R}^{3}$ (cf. [55, 56, 54, 22]).

Originally, the model of Perona and Malik [50] was developed to anisotropically diffuse gray values $u_{0}: \Omega \rightarrow \mathbb{R}$, i.e., $u: \Omega_{T} \rightarrow \mathbb{R}$ solves for $u(0, \cdot)=u_{0} \in L^{\infty}(\Omega)$, and all $0<t \leqslant T$,

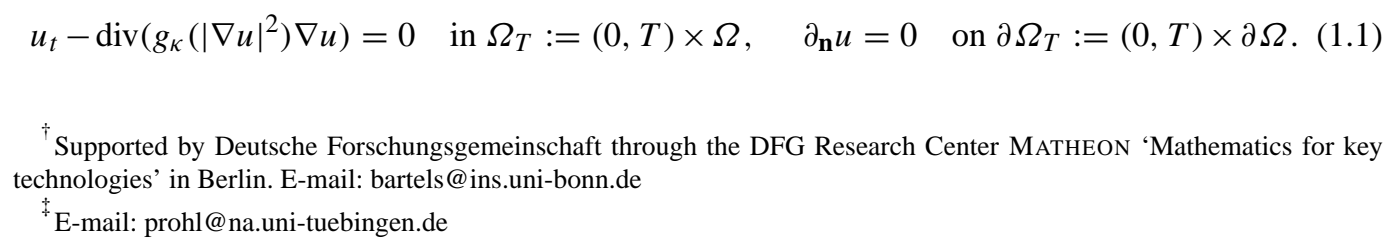


Let $\Omega \subset \mathbb{R}^{M}$ be a bounded, Lipschitz domain. A standard example of a smooth nonincreasing positive function $g_{\kappa}: \mathbb{R} \rightarrow \mathbb{R}$ with $g_{\kappa}(0)=1$ and $s g_{\kappa}\left(s^{2}\right) \rightarrow 0$ at infinity is $g_{\kappa}\left(s^{2}\right)=$ $\left(1+s^{2} / \kappa\right)^{-1}, \kappa>0$. Problem (1.1) may then formally be considered as the $L^{2}$-gradient flow related to the nonconvex, noncoercive energy $G_{\kappa}(u)=\kappa \int_{\Omega} \phi_{\kappa}\left(|\nabla u|^{2}\right) \mathrm{d} \mathbf{x}$ with $\phi_{\kappa}^{\prime}\left(s^{2}\right)=s g_{\kappa}\left(s^{2}\right)$ and $\phi_{\kappa}\left(s^{2}\right)=\frac{1}{2} \log \left(1+s^{2} / \kappa\right)$. In the following, we drop the subscript $\kappa>0$ in case $\kappa=1$. The motivation for this model is to suppress diffusion at regions of large gradients to preserve sharp edges; it is in these regions where the gradient exceeds some threshold $0<z=z(\kappa)$, so that 1.1 becomes backward parabolic, whereas 1.1 is forward parabolic elsewhere. As a consequence of the additional noncoercivity, well-posedness of (1.1) is a delicate matter [45, 44, 38], and recent literature mainly focuses on modified versions of [1.1]. These are partly outlined in [45], together with the proposal of a 'reasonable' concept of solutions: in [27, 33], solutions for a spatial discretization $(h>0)$ are characterized $(M=1)$, and a system of PDE's coupled via nonlinear boundary conditions is derived to control the limiting function admitting jumps as $h \rightarrow 0$ in case $\kappa=\mathcal{O}\left(h^{-1}\right)$ at all times (see also [14]). These ansatzes are mainly motivated to construct global solutions with possible (spatial) discontinuities, which enjoy further properties, like e.g. decrease of energy and maximum principle. Another strategy introduces finite scales to the problem by spatial [19] or temporal [3, 49, 10] convolution of entries of $g_{\kappa}$ to verify (local/global) existence of Sobolev type solutions. A third, closely related approach adds regularizing terms, like $+\varepsilon \Delta^{2} u$ or $-\varepsilon \Delta u_{t}, \varepsilon>0$, to $(1.1)$ to allow for weak solutions $u^{\varepsilon}: \Omega_{T} \rightarrow \mathbb{R}$, and derive governing PDE's for appropriate limits (as $\varepsilon \rightarrow 0$ ), which are of bounded variation to ensure edge-preserving regularization (cf. [11]).

The growth condition for the above functional at infinity is a crucial feature to keep and enhance edges and corners of input images. In [51], the total variation functional $J(u)=\int_{\Omega}|\nabla u| \mathrm{d} \mathbf{x}$ is introduced to denoise and restore inputs $u_{0}: \Omega \rightarrow \mathbb{R}$, and the $L^{2}$-gradient flow formally reads

$$
u_{t}-\operatorname{div}\left(\frac{\nabla u}{|\nabla u|}\right)=0 \quad \text { in } \Omega_{T}, \quad \partial_{\mathbf{n}} u=0 \quad \text { on } \partial \Omega_{T} .
$$

Advantages of this model over (1.1) are well-posedness in the practically relevant space of functions with bounded variation, and further analytical [4, 5] and numerical [1, 25, 21, 32, 31, 20] understanding. However, computational experiments for a stable discretization in Section 2.2 evidence improved flexibility of the model of Perona-Malik over the $T V$-model, due to the asymptotic sublinear growth of related energies: fading away of bulk features at logarithmic timescale (in contrast to linear one), or invariance of nonconvex supports (in contrast to variance of nonconvex supports); see also [42, 31], and Figure 2.3 below.

Processing colored images is a more complex task, where brightness and directions/chromaticity of an RGB vector field $\mathbf{u}_{0}: \Omega \rightarrow \mathbb{R}^{3}$ are independently and anisotropically diffused [54, 56]: the scalar brightness $\left|\mathbf{u}_{0}\right|: \Omega \rightarrow \mathbb{R}$ is regularized by $(1.1)$ or 1.2 , for example, whereas the $p$ harmonic map heat flow to the sphere $\mathbb{S}^{2}$, for $1 \leqslant p \leqslant 2$, is used to regularize directions $\mathbf{u}_{0} /\left|\mathbf{u}_{0}\right|$ : $\Omega \rightarrow \mathbb{S}^{2}$ (cf. [41, 36, 3, 18, 6, 8], and references therein for analytical and numerical results for $p$-harmonic map heat flow). In particular, computational studies in [6, 9] evidence an interesting dynamical behavior for cases $1<p \leqslant 2$, like finite-time blow-ups, or geometric changes. In this paper we continue this program by numerically studying anisotropic diffusion of directions by a vectorial Perona-Malik model, where $\mathbf{u}: \Omega_{T} \rightarrow \mathbb{R}^{3}$ solves

$$
\mathbf{u}_{t}-\mathbf{u} \times(\mathbf{u} \times \boldsymbol{D} \boldsymbol{G}(\mathbf{u}))=0 \quad \text { in } \Omega_{T}, \quad \partial_{\mathbf{n}} \mathbf{u}=0 \quad \text { on } \partial \Omega_{T}, \quad \mathbf{u}(0, \cdot)=\mathbf{u}_{0} \quad \text { in } \Omega,
$$


for $\mathbf{u}_{0}: \Omega \rightarrow \mathbb{S}^{2}$, and $(\boldsymbol{D G}(\mathbf{u}), \mathbf{v})=\int_{\Omega} g\left(|\nabla \mathbf{u}|^{2}\right)\langle\nabla \mathbf{u}, \nabla \mathbf{v}\rangle$ d $\mathbf{x}$. Again, solvability of this model is a critical issue, and related problems definitely exceed those mentioned before in the context of the scalar problem (1.1); in this paper, we focus on numerical aspects, i.e., construction and analysis of fully discrete versions of (1.3), and their iterative solution. This is a nontrivial task due to the sphere constraint and nonconvexity of the underlying potential.

In the following, let $\mathbf{V}_{h}:=\left[V_{h}\right]^{3}$ be the space of finite element functions, which are elementwise affine and globally continuous, i.e., $V_{h}=\left\{v_{h} \in C(\bar{\Omega}):\left.v_{h}\right|_{K}\right.$ is affine $\left.\forall K \in \mathcal{T}_{h}\right\}$, where $\mathcal{T}_{h}$ is a triangulation of $\Omega$. Further, $I_{k}:=\left\{t_{m}\right\}_{m} \geqslant 0$ denotes an equidistant net of mesh size $k>0$ to discretize $[0, T]$. We study, both theoretically and numerically, properties of iterates $\left\{\mathbf{U}^{m}\right\} \subset \mathbf{V}_{h}$ of Algorithm 1.1 below to motivate properties of certain limits of approximating sequences $\{\overline{\mathcal{U}}\}_{k, h}$, where $\overline{\mathcal{U}} \in C\left([0, T] ; \mathbf{V}_{h}\right)$ is defined by

$$
(t, \mathbf{x}) \mapsto \overline{\mathcal{U}}(t, \mathbf{x}):=\frac{t-t_{m}}{k} \mathbf{U}^{m+1}(\mathbf{x})+\frac{t_{m+1}-t}{k} \mathbf{U}^{m}(\mathbf{x}), \quad t \in\left[t_{m}, t_{m+1}\right) .
$$

This scheme is constructed to satisfy a discrete sphere constraint, i.e. $\mathbf{U}^{m+1}(\mathbf{x}) \in \mathbb{S}^{2}$ at the nodes of the triangulation $\mathcal{T}_{h}(m \geqslant 0)$, and a discrete energy law (see Lemma 3.1).

Let $d_{t} \mathbf{U}^{m+1}:=k^{-1}\left(\mathbf{U}^{m+1}-\mathbf{U}^{m}\right)$ and $\overline{\mathbf{U}}^{m+1 / 2}:=\frac{1}{2}\left(\mathbf{U}^{m+1}+\mathbf{U}^{m}\right)$ for $m \geqslant 1$; we use a discrete version (reduced integration) of the inner product in $L^{2}\left(\Omega ; \mathbb{R}^{3}\right)$, i.e., $(\mathbf{f}, \mathbf{g})_{h}=\int_{\Omega} \mathcal{I}_{h}\langle\mathbf{f}, \mathbf{g}\rangle \mathrm{d} \mathbf{x}$, for the nodal interpolation operator $\mathcal{I}_{h}$, and $\mathbf{f}, \mathbf{g} \in C\left(\bar{\Omega} ; \mathbb{R}^{3}\right)$; we refer to Section 2.2 for further details regarding notation.

Algorithm 1.1 For $m \geqslant 0$, let $\mathbf{U}^{m} \in \mathbf{V}_{h}$, and determine $\left(\mathbf{U}^{m+1}, \boldsymbol{L}^{m+1}\right) \in\left[\mathbf{V}_{h}\right]^{2}$ from

$$
\begin{aligned}
& \left(d_{t} \mathbf{U}^{m+1}, \boldsymbol{\psi}\right)_{h}-\left(\overline{\mathbf{U}}^{m+1 / 2} \times\left(\overline{\mathbf{U}}^{m+1 / 2} \times \boldsymbol{L}^{m+1}\right), \boldsymbol{\psi}\right)_{h}=0 \quad \forall \boldsymbol{\psi} \in \mathbf{V}_{h}, \\
& \left(\boldsymbol{L}^{m+1}, \boldsymbol{\psi}\right)_{h}=\left(\widetilde{\gamma}\left(\nabla \mathbf{U}^{m+1}, \nabla \mathbf{U}^{m}\right), \nabla \boldsymbol{\psi}\right) \quad \forall \boldsymbol{\psi} \in \mathbf{V}_{h},
\end{aligned}
$$

with $\tilde{\boldsymbol{\gamma}}(\boldsymbol{\alpha}, \boldsymbol{\beta})=\left(\widetilde{\gamma}\left(\alpha_{i j}, \beta_{i j}\right)\right)_{i j} \in \mathbb{R}^{3 \times M}$ for $\boldsymbol{\alpha}=\left(\alpha_{i j}\right)_{i j}, \boldsymbol{\beta}=\left(\beta_{i j}\right)_{i j} \in \mathbb{R}^{3 \times M}$, where

$$
\tilde{\gamma}(a, b)= \begin{cases}\frac{\phi\left(a^{2}\right)-\phi\left(b^{2}\right)}{a-b} & \text { if } a \neq b, \\ g\left(a^{2}\right) a & \text { if } a=b .\end{cases}
$$

Here, the function $\boldsymbol{L}^{m+1}$ is an approximation of $\boldsymbol{D} \boldsymbol{G}(\mathbf{u})$. For practical purposes, a simple fixed point scheme together with a stopping criterion (Algorithm 3.1 ) is used to approximate solutions to Algorithm 1.1 As is stated in Theorem 3.1, iterates still satisfy the sphere constraint, and converge to solutions of Algorithm 1.1 provided that $k \leqslant C h^{2}$.

The remainder of this paper is as follows: Section 2.1 surveys recent strategies to solve 1.1); a full discretization of 1.1 is proposed in Section 2.2 where solutions satisfy a discrete energy law $(M \geqslant 1)$; in order to exclude additional spatio-temporal perturbation effects, which may affect dynamics of staircasing [45] at finite scales, we consider a direct discretization of (1.1) (rather than regularized versions of (1.1)). Comparative computational experiments for the total variation flow and the Perona-Malik evolution show very different quantitative behaviors. In Section 3 , we study the (fully discrete) Perona-Malik evolution into the sphere, and prove its stability; computational studies show different dynamics in comparison with regularized 1-harmonic map heat flow. 


\section{Gradient flow of a nonconvex functional: the scalar case}

Nonstationary equations

$$
u_{t}=-D F(u) \quad \text { in } \Omega_{T}, \quad \partial_{\mathbf{n}} u=0 \quad \text { on } \partial \Omega_{T}, \quad u(0, \cdot)=u_{0} \quad \text { in } \Omega,
$$

for nonconvex $F(u)=\int_{\Omega} \psi(|\nabla u|) \mathrm{d} \mathbf{x}$ are forward-backward parabolic, which in the case of coercivity of $\psi: \mathbb{R} \rightarrow \mathbb{R}$ possess global Young measure solutions [24]. Such solutions are typically not unique; however, the first moment of the Young measure is unique, and its stability is reflected by maximum and comparison principles, as well as an energy decay property. Numerical experiments in [30] give further insight into dynamics of 2.1] in the coercive case: (i) the gradient flow for the convexified density (e.g., no 'wrinkling', see below) does not approximate the above Young measure solution of 2.1]; (ii) formation of wrinkles of size of spatial discretization $h>0$ only appears in the locally concave regions $L U S=\left\{s \in \mathbb{R}: \psi^{\prime \prime}(s)<0\right\}$ of the energies, and not in the larger set of globally unstable regions $G U S=\left\{s \in \mathbb{R}: \psi(s)>\psi^{* *}(s)\right\}$, and movement of 'wrinkling regions' at later times is observed.

The properties of 2.1 for nonconvex, noncoercive $\psi$ are much less understood, and the approach in [24] to construct Young-measure solutions via Rothe's method for the above setup does not apply here. Recently [53], the construction of Young-measure solutions to (1.1) for $M=1$ succeeded, which is based on a reformulation of the problem; it is pointed out in [53] that the solutions constructed suffer from severe instabilities, which e.g. occur in the evolution for 'mixed' initial data $u_{0}$, where $u_{0, x}$ belongs to both convex and concave regions of $\psi$. Discretization of the governing PDE, or approximation respectively regularization of $F$ are common strategies to obtain well-posed problems, to construct and identify possible limits to control asymptotical dynamics of the regularized model. The work [37] is an interesting example for this program, where a notion of gradient flow of the nonconvex Mumford-Shah functional $\mathcal{F}: S B V(\Omega) \rightarrow \mathbb{R}(M=1)$ is introduced as the limit of the well-posed gradient flow $(h>0)$

$$
u_{t}^{h}=-D \hat{F}_{h}\left(u^{h}\right) \quad \text { in } \Omega_{T}, \quad u_{h}(0, \cdot)=u_{0, h},
$$

for the functional $\hat{F}_{h}: P C_{h}^{2} \rightarrow \mathbb{R}$,

$$
\hat{F}_{h}(u)=\frac{1}{h} \int_{\mathbb{R}} \arctan \left(\frac{|u(x+h)-u(x)|^{2}}{h}\right) \mathrm{d} x,
$$

with piecewise constant functions $P C_{h}^{2}:=\left\{u \in L^{2}(\mathbb{R}):\left.u\right|_{K} \in P_{0}\left(K_{i}\right)\right\}$, and which converge to $\mathcal{F}$ in variational sense. Here, a nonoverlapping covering $\bigcup_{i} \overline{K_{i}}=\mathbb{R}$ is used, which consists of open triangles such that $K_{i} \cap K_{j}=\emptyset$ for $0 \leqslant i \neq j$, with area $\sup _{i}\left|K_{i}\right| \leqslant h$; it is shown in [37] that well-defined limits $u: \Omega_{T} \rightarrow \mathbb{R}$ solve local heat equations with homogeneous Neumann boundary conditions at (a finite number of) starting discontinuities, which keep position and exist for finite ('merging') or infinite times ('surface energy monotonicity' $\frac{\mathrm{d}}{\mathrm{d} t} \mathcal{H}^{0}\left(S_{u(t, \cdot)}\right) \leqslant 0$, i.e., the singularity set $S_{u(t, \cdot)}:=\left\{x \in \mathbb{R}: u^{+}(t, x) \neq u^{-}(t, x)\right\}$ is nonincreasing; see e.g. [4] $)$, and enjoys desirable properties, like maximum principle, energy decay $\frac{\mathrm{d}}{\mathrm{d} t} \mathcal{F}(u(t, \cdot)) \leqslant 0$, and Hölder continuity in time. Due to some connection between Mumford-Shah and Perona-Malik problems, those results may serve as a motivation to better understand the latter (cf. [43], and the literature cited therein).

Another example for unexpected dynamics which follows a similar program is studied in [14] for the nonconvex $\phi(\xi)=\min \left\{\xi^{2}, 1\right\}(M=1)$, where (nonunique) limits of restricted 
functions of bounded variation $\left\{u_{h}(t, \cdot)\right\} \subset V_{h} \subset B V(\Omega)$ (which solve a problem similar to (2.2)) are in $L^{\infty}([0, \infty), B V(0,1)) \cap A C^{2}\left([0, \infty) ; L^{2}(0,1)\right)$, and satisfy (i) a free boundary value problem (local linear heat equations, with homogeneous Neumann conditions at interfaces), (ii) a maximum principle (no comparison principle), and (iii) monotonicity for the total variation, i.e. $|D u(t, \cdot)|((0,1)) \leqslant\left|D u_{0}\right|((0,1))$ for $t \geqslant 0$; moreover, the discontinuity set $S_{u(t, \cdot)}$ may move and subsets may merge in space-time. Part of these results have recently been extended to PeronaMalik $(M=1)$ in [15]; in particular, additional temporal discretization effects are shown to play a crucial role for limiting solutions $(k, h \rightarrow 0)$, unless $k \leqslant C \exp \left(-1 / h^{\alpha}\right)$ for $\alpha>2$ (see [15, Props. $6.1,6.2])$.

\subsection{Scalar Perona-Malik-an overview}

Analytical studies [5] of the scalar-valued total variation $(T V)$ flow $(p=1)-u_{t} \in \partial J(u)$, for $u(0, \cdot)=u_{0} \in L^{2}(\Omega)$, and $J(u(t, \cdot))=|D u(t, \cdot)|(\Omega)$ show interesting characterizations of the strong solution in the sense of semigroup theory: (i) finite extinction time $(M=2)$, (ii) $u(t, \cdot) \in$ $L^{\infty}(\Omega)$ for $t>0$ if $u_{0} \in L^{M}(\Omega)$, and no $L^{1}-L^{2}$-regularizing effect for $L^{1}(\Omega)$-initial data in general, (iii) $C^{1, \alpha}$-regularity of level sets $\partial^{*}[u(t, \cdot)>\lambda]$ for $u_{0} \in L^{M}(\Omega)$ of decreasing size, i.e., $\frac{\mathrm{d}}{\mathrm{d} t} \mathcal{H}^{M-1}\left(\partial^{*}[u(t, \cdot)>\lambda]\right) \leqslant 0$, and (iv) invariance of supports, provided e.g. the curvature of the smooth boundary of the simply connected convex domain is not too large. The more practical notion of weak solutions is established and studied in [40, 32], and convergent finite element methods are discussed in [32, 31]. Consider $\bar{U}^{\varepsilon, h, k}(t, \cdot):=\frac{t-t_{m-1}}{k} U^{m}+\frac{t_{m}-t}{k} U^{m-1} \in V_{h}$ in $t \in\left[t_{m-1}, t_{m}\right)$, where the iterates $\left\{U^{m}\right\} \subset V_{h}$ solve the regularized, fully discrete problem

$$
\left(d_{t} U^{m}, v_{h}\right)+\left(\frac{\nabla U^{m}}{\sqrt{\left|\nabla U^{m}\right|^{2}+\varepsilon^{2}}}, \nabla v_{h}\right)=0 \quad \forall v_{h} \in V_{h} .
$$

The iterates satisfy a discrete energy law, and convergence of $\left\{\bar{U}^{\varepsilon, h, k}\right\} \subset C\left([0, T] ; V_{h}\right)$ towards strong solutions of the $T V$-problem with a rate

$$
\left\|u-\bar{U}^{\varepsilon, h, k}\right\|_{L^{\infty}\left(0, T ; L^{2}\right)} \leqslant C \sqrt{|\Omega| T} \sqrt{\varepsilon}+C_{1}(\varepsilon) k+C_{2}(\varepsilon) h^{2}
$$

is verified in [31, 32] provided that the underlying triangulation is quasiuniform, and $k=\mathcal{O}\left(h^{2}\right)$. Moreover, the constants $C_{i}(\varepsilon)>0$ depend on $\varepsilon^{-1}$ in a low polynomial order.

A mathematical study of Perona-Malik's model (1.1) started with Kichenassamy's work [45], where weak solutions for (1.1) were excluded, and a concept of generalized solutions is motivated, which allows for (energetically favorable) step functions in finite and infinite time, to analytically describe numerically observed formation, merging, and segmentation $(t \rightarrow \infty)$ during a 'staircasing' process (cf. also Figure 2.1 below). The following general assumptions from [45] for $g_{\kappa}: \mathbb{R} \rightarrow \mathbb{R}$ apply to the upper prototypic example, in particular.

ASSUMPTION $2.1 \quad$ (i) $g_{\kappa}(\xi)>0$ for all $\xi \geqslant 0$.

(ii) The parameter $\kappa>0$ defines a positive critical value $z(\kappa)$ such that $\partial_{\xi}\left(\xi g_{\kappa}\left(\xi^{2}\right)\right)>0$ for $|\xi|<z(\kappa)$, and $\partial_{\xi}\left(\xi g_{\kappa}\left(\xi^{2}\right)\right)<0$ for $|\xi|>z(\kappa)$.

(iii) Both $g_{\kappa}(\xi)$ and $\partial_{\xi}\left(\xi g_{\kappa}\left(\xi^{2}\right)\right)$ tend to zero as $\xi$ goes to infinity.

In Esedoglu's work [27], solutions $u_{h}: \Omega_{T} \rightarrow \mathbb{R}$ for a spatial semi-discretization of (1.1) in the case $\kappa=\kappa(h)$ are shown to converge $(h \rightarrow 0)$ to solutions of the following system of heat 
equations coupled to each other through nonlinear boundary conditions that may become singular at finite times $(M=1)$ :

$$
\frac{\partial u_{i}}{\partial t}=\frac{\partial^{2} u_{i}}{\partial x^{2}} \quad \text { in }\left(p_{i-1}, p_{i}\right), \quad \frac{\partial u_{i}}{\partial x}\left(t, p_{i}\right)=\frac{\partial u_{i+1}}{\partial x}\left(t, p_{i}\right)=\frac{1}{u_{i+1}\left(t, p_{i}\right)-u_{i}\left(t, p_{i}\right)},
$$

for all $1 \leqslant i \leqslant N-1$, and $\frac{\partial u_{1}}{\partial x}\left(t, p_{0}\right)=\frac{\partial u_{N}}{\partial x}\left(t, p_{N}\right)=0$. Here, $p_{0}<p_{1}<\cdots<p_{N}$ denote the positions of jumps, which are shown not to move during the evolution process, but will vanish within finite time (energy argument). (Changes in time of the jump set for $M=2$ is computationally evidenced in [28].) Locally existing solutions satisfy the maximum principle (but no comparison principle, [27]), are Hölder continuous in time, and decrease the energy

$$
P M_{E}(t):=\frac{1}{2} \sum_{i=1}^{N} \int_{p_{i-1}}^{p_{i}}\left|\frac{\partial u_{i}}{\partial x}(t, \cdot)\right|^{2} \mathrm{~d} x+\sum_{i=1}^{N-1} \log \left(\left|u_{i+1}\left(t, p_{i}\right)-u_{i}\left(t, p_{i}\right)\right|\right),
$$

which, however, is not bounded from below as jumps tend to zero. Canonical continuation of solutions beyond blow-ups at 'quenching times' is possible; this reflects merging of neighboring terraces ('coarsening') in the final step during evolution, which is preceded by the formation of terraces ('staircasing'). Hence, this program follows the proposal [45] to explain formation and merging of terraces; see [27] for illustrative simulations in 1D; see also 1D-simulations respectively 2D-simulations in [27] respectively [46, pp. 84ff], [43].

A different line of research uses recent results [4, 18] about $\Gamma$-convergent approximations of energies $F: B V(\Omega) \rightarrow \mathbb{R}$ of the form [48]

$$
F(u)=\int_{\Omega} f(|\nabla u|) \mathrm{d} \mathbf{x}+\int_{S_{u}} \varphi\left(u^{+}-u^{-}\right) \mathrm{d} \mathcal{H}^{M-1}+C\left|D^{c} u\right|
$$

by sequences $F_{\varepsilon}: W^{2,2}(\Omega) \rightarrow \mathbb{R}$,

$$
F_{\varepsilon}(u)=\int_{\Omega} f_{\varepsilon}(|\nabla u|) \mathrm{d} \mathbf{x}+|r(\varepsilon)|^{3} \int_{\Omega}\left|\nabla^{2} u\right|^{2} \mathrm{~d} \mathbf{x}
$$

Here, $C_{1}(\sqrt{z}-1) \leqslant \varphi(z) \leqslant C_{2}(z+1)$ for all $z \geqslant 0$, and $C_{1}, C_{2}>0$ (which excludes the Mumford-Shah functional; cf. [17] for $M=1$ ), and $\left|D^{c} u\right|$ denotes the total variation of the Cantor part of the measure $D u$; moreover, $\left\{f_{\varepsilon}\right\}$ is any family of positive, nondecreasing functions of nonconvex or convex-concave shape, and $f$ respectively $\varphi$ are defined as proper limits of $f_{\varepsilon}$ respectively $r(\varepsilon) f_{\varepsilon}(\cdot / r(\varepsilon))$. As is pointed out in [48], by using the right rescaling $r(\varepsilon)=\varepsilon / \log (1 / \varepsilon)$ for the Perona-Malik functional, we have

$$
G_{\varepsilon}(u)+\left(\frac{\varepsilon}{\log \frac{1}{\varepsilon}}\right)^{3} \int_{\Omega}\left|\nabla^{2} u\right|^{2} \mathrm{~d} \mathbf{x} \stackrel{\Gamma}{\rightarrow} \int_{\Omega}|\nabla u|^{2} \mathrm{~d} \mathbf{x}+c \int_{S_{u}}\left|u^{+}-u^{-}\right|^{1 / 2} \mathrm{~d} \mathcal{H}^{M-1},
$$

for some computable $c>0$. Then the general results in [48] allow constructing generalized PeronaMalik equations, where solutions of formal $L^{2}$-gradient flows to 2.4) are interpreted as limits (as $\varepsilon \rightarrow 0$ ) of existing $L^{2}$-gradient flows of [2.5); in [11, 12], the authors study the $L^{2}$-gradient flow for the $\Gamma$-limit of the slightly different scaling (cf. 11.3$) \mathcal{F}_{v}: W^{2,2}((0,1)) \rightarrow \mathbb{R}$,

$$
\mathcal{F}_{v}(u):=\frac{1}{2} \int_{0}^{1}\left(\frac{\phi\left(u_{x}\right)}{v \phi(1 / v)}+v^{3}\left|u_{x x}\right|^{2}\right) \mathrm{d} x \quad(v>0),
$$


which is proved to be $\mathcal{F}: \mathcal{P}((0,1)) \rightarrow \mathbb{R}$ with

$$
\mathcal{F}(u):=2 \sqrt{\frac{2}{3}} \sum_{x \in S_{u}}\left|u^{+}(x)-u^{-}(x)\right|^{1 / 2} \quad \text { for } \quad \mathcal{P}((0,1)):=\left\{u \in S B V((0,1)): D^{a} u=0\right\},
$$

where $D^{a} u$ denotes the absolutely continuous part of the derivative $D u$; a motivation for the scaling in 2.6. comes from studying solutions of the $L^{2}$-gradient flow (for $\Omega=(0,1)$ )

$$
u_{t}^{\varepsilon}+\frac{1}{2}\left(\phi^{\prime}\left(u_{x}^{\varepsilon}\right)\right)_{x}+\varepsilon^{2} u_{x x x x}^{\varepsilon}=0 \quad \text { in } \Omega_{T}, \quad u_{x}^{\varepsilon}=u_{x x x}^{\varepsilon}=0 \quad \text { on } \partial \Omega_{T}
$$

at large times $t=\mathcal{O}\left(1 / \nu \phi(1 / v)\right.$ ), with $\varepsilon^{2}=v^{4} \phi(1 / v)$, where coarsening takes place (after an initial period of rapid formation of microstructures, followed by a longer coarsening period). Then the $L^{2}$-gradient dynamics for $\mathcal{F}$ that is identified with the global $L^{2}$-minimizing movement in the sense of De Giorgi leads to a coupled system of nonlinear ODE's, which controls dynamics of local heights of the piecewise constant function $\mathcal{P}((0,1)) \ni u(t, \cdot)=s_{0}+\sum_{j=1}^{N} s_{j}(t) \chi_{\left[p_{j}, 1\right)}$; again, initial places of jumps do not move, and merging is admitted; see also [30, Example 1] for computational evidence and discussion of formation and coarsening of piecewise constant mappings at times $t>0$ for cases $\left\{u_{0, x} \in L U S\right\} \neq \emptyset$.

Finally, we mention [33], where results help to better compare $T V$-flow and 1.1], and illustrate different behaviors of (1.1) in different spatial dimensions $(M \geqslant 1)$ : for $M=1$, the total variation of solutions to 1.1 is shown to be nonincreasing in time, while counterexamples show that this assertion fails for $M=2$. In addition, the authors in [33] study solutions to a semidiscretization in space for $M=1,2$, where only maximum and energy decay property are validated $(M=1)$, due to failure of tools which apply only for the continuous model (1.1), like the chain rule, for example. An interesting recent result $(M=1)$ is given in [34], which shows that instantaneous staircasing may not always be expected for 'mixed'/transcritical data: it is shown that the set of initial data for which Perona-Malik has a local in time classical solution $u: \Omega_{T} \rightarrow \mathbb{R}$ is dense in $C^{1}(\Omega)$.

Computational experiments for (1.1) nourish the above analytical studies; unfortunately, it is difficult to design fully discrete schemes that satisfy all properties mentioned above at the same time, like energy, positivity, and maximum principle for $M \geqslant 2$, and not evident how to properly relate numerical discretization parameters [15]. In [28], Esedoglu verifies a restricted comparison principle for a spatial semidiscretization $(M \geqslant 2)$, with additional control over gradients in the case $M=1$; computational experiments are provided to evidence failure of these properties for $M=2$. In [10], a discrete maximum principle is established for a fully discrete finite difference scheme to solve Nitzberg-Shiota's [49] temporal regularization of $(1.1)(M=2)$. Unconditional convergence in $C\left(0, T ; L^{2}(\Omega)\right) \cap L^{2}\left(0, T ; W^{1,2}(\Omega)\right)$ of the whole sequence $\left\{\bar{U}^{h, k}\right\}$ (see construction as before in (2.3) of solutions $\left\{U^{m}\right\}$ of a semi-implicit finite element discretization $(M=2)$ for the regularized version [19] with smoothing kernel

$$
\mathcal{G}_{\sigma}(x)=\frac{1}{4 \pi \sigma} \exp \left(-\frac{|x|^{2}}{4 \sigma}\right), \quad \sigma>0,
$$

in the form

$$
\left(d_{t} U^{m}, v_{h}\right)+\left(g\left(\left|\nabla \mathcal{G}_{\sigma} * U^{m-1}\right|^{2}\right) \nabla U^{m}, \nabla v_{h}\right)=0 \quad \forall v_{h} \in V_{h}, \quad m \geqslant 1,
$$


for $u^{0}=u_{0} \in L^{2}(\Omega)$ to a unique limit which solves its continuous version is verified in [42]: its proof rests upon the (a priori) bound

$$
\frac{1}{2}\left\|U^{m}\right\|_{L^{2}}^{2}+\frac{k^{2}}{2} \sum_{j=1}^{m}\left\|d_{t} U^{j}\right\|_{L^{2}}^{2}+k \sum_{j=1}^{m} \int_{\Omega} \alpha_{j-1}\left|\nabla U^{j}\right|^{2} \mathrm{~d} \mathbf{x}=\frac{1}{2}\left\|u_{0}\right\|_{L^{2}}^{2}, \quad m \geqslant 1,
$$

with $\alpha_{m-1}:=g\left(\left|\nabla \mathcal{G}_{\sigma} * U^{m-1}\right|\right) \geqslant v_{\sigma}>0$. The properties of $g\left(\left|\nabla \mathcal{G}_{\sigma} * \cdot\right|\right)$, together with equation (2.8), are key tools for a compactness argument, and to establish solution character of a proper limit in $L^{2}\left(\Omega_{T}\right)$ for the continuum version of (2.7). Interesting computations are also included in [42] for values $h=k \approx 10^{-3} \operatorname{div} 10^{-2}$, and $\sigma \approx 10^{-8} \operatorname{div} 10^{-5}$, where cusp-like edges are preserved/enhanced, while gross parts of the solution are smoothened. A corresponding program was realized for a semi-implicit finite volume scheme in [47]; more recently, suboptimal rates $\left\|\bar{U}^{h, k}-u\right\|_{L^{2}\left(\Omega_{T}\right)} \leqslant C_{T} \sqrt{h}$ are verified for the case $k=h$ in [39], using a (discrete) Gronwall argument.

Long-time dynamics of a fully discrete version of [1.1] is studied in the recent work [26]. Here, a semi-implicit discretization based on finite differences for a reformulation of (1.1) is used, and a discrete energy inequality is derived to study asymptotic behavior of iterates $\left\{U^{m}\right\}$ for $m \rightarrow \infty$.

The works [42, 47, 39] employ properties of the special regularization of the elliptic PDE (1.1) given in [19]; in Section 2.2, we consider a full, finite element based discretization of the original problem 1.1) which exploits its character as gradient flow for the nonconvex functional $G$, for $M \geqslant 2$, and whose iterates $\left\{U^{m}\right\}$ satisfy the discrete energy law

$$
k \sum_{j=1}^{m}\left\|d_{t} U^{j}\right\|_{L^{2}}^{2}+G\left(U^{m}\right)=G\left(u_{0}\right), \quad m \geqslant 1 \quad(\kappa>0) .
$$

To reach this goal, the Fréchet derivative of $G_{\kappa}: V_{h} \rightarrow \mathbb{R}$ is replaced by a difference quotient in Algorithm 2.1. As outlined above, convergence behavior remains unclear, but may easily be concluded - based on the discrete energy identity [2.9] - for regularizations of (1.1).

\subsection{Scalar Perona-Malik equation-energy decreasing discretization}

We propose a stable, implicit finite element discretization of a slightly modified version of (1.1) $(M=2)$; a discrete energy law is established for an implicit discretization in time. Numerical simulations are performed to directly compare properties with total variation flow. This section also serves as a preparatory step and motivation for the scheme devised in Section 3 . We follow [50, 27, 28] and evolve $u_{0} \in L^{\infty}(\Omega)$ via $(M=2)$

$$
u_{t}-\operatorname{div}\left(\begin{array}{l}
g\left(u_{x}^{2}\right) u_{x} \\
g\left(u_{y}^{2}\right) u_{y}
\end{array}\right)=0 \quad \text { in } \Omega_{T}, \quad \partial_{\mathbf{n}} u=0 \quad \text { on } \partial \Omega_{T} .
$$

Notice that 2.10 is formally the gradient flow to $\bar{G}(u)=\int_{\Omega}\left(\phi\left(u_{x}^{2}\right)+\phi\left(u_{y}^{2}\right)\right) \mathrm{d} x \mathrm{~d} y$.

Let $\mathcal{T}_{h}$ be a regular triangulation of the polygonal or polyhedral bounded Lipschitz domain $\Omega \subset \mathbb{R}^{M}$ into triangles or tetrahedra of maximal diameter $h>0$ for $M=2$ or $M=3$, respectively. Given the set of all nodes (or vertices) $\mathcal{N}$ in $\mathcal{T}_{h}$ and letting $\left\{\varphi_{\mathbf{z}}: \mathbf{z} \in \mathcal{N}\right\}$ denote the nodal basis 
in $V_{h}$, we define the nodal interpolation operator $\mathcal{I}_{h}: C(\bar{\Omega}) \rightarrow V_{h}$ by $\mathcal{I}_{h} \psi:=\sum_{\mathbf{z} \in \mathcal{N}} \psi(\mathbf{z}) \varphi_{\mathbf{z}}$ for $\psi \in C(\bar{\Omega})$. For $\phi, \psi \in C(\bar{\Omega})$, a discrete inner product is defined by

$$
(\phi, \psi)_{h}:=\int_{\Omega} \mathcal{I}_{h}(\phi \psi) \mathrm{d} \mathbf{x}=\sum_{\mathbf{z} \in \mathcal{N}} \beta_{\mathbf{z}} \phi(\mathbf{z}) \psi(\mathbf{z}),
$$

where $\beta_{\mathbf{z}}=\int_{\Omega} \varphi_{\mathbf{z}} \mathrm{d} \mathbf{x}$ for all $\mathbf{z} \in \mathcal{N}$; we define $\|\psi\|_{h}^{2}:=(\psi, \psi)_{h}$. We remark that

$$
\left\|\psi_{h}\right\|_{L^{2}} \leqslant\left\|\psi_{h}\right\|_{h} \leqslant(M+2)^{1 / 2}\left\|\psi_{h}\right\|_{L^{2}}
$$

for all $\psi_{h} \in V_{h}$. Basic interpolation estimates yield

$$
\left|\left(\phi_{h}, \psi_{h}\right)_{h}-\left(\phi_{h}, \psi_{h}\right)\right| \leqslant C h\left\|\phi_{h}\right\|_{L^{2}}\left\|\nabla \psi_{h}\right\|_{L^{2}}
$$

for all $\phi_{h}, \psi_{h} \in V_{h}$;

Algorithm 2.1 For $J \geqslant 0$, and given $\left\{U^{j}\right\}_{0 \leqslant j \leqslant J} \subset V_{h}$, determine $U^{J+1} \in V_{h}$ from

$$
\left(d_{t} U^{J+1}, \psi\right)_{h}+\left(\tilde{\gamma}\left(\nabla U^{J+1}, \nabla U^{J}\right), \nabla \psi\right)=0 \quad \forall \psi \in V_{h},
$$

where $\tilde{\gamma}(\boldsymbol{\alpha}, \boldsymbol{\beta})=\left(\widetilde{\gamma}\left(\alpha_{1}, \beta_{1}\right), \widetilde{\gamma}\left(\alpha_{2}, \beta_{2}\right)\right)^{T} \in \mathbb{R}^{2}$ for $\boldsymbol{\alpha}=\left(\alpha_{1}, \alpha_{2}\right), \boldsymbol{\beta}=\left(\beta_{1}, \beta_{2}\right)$, with $\widetilde{\gamma}(\cdot, \cdot)$ from Algorithm 1.1 .

The algorithm for the approximation of scalar Perona-Malik flow is stated for two space dimensions $(M=2)$ but can easily be modified for arbitrary $M$. The use of reduced integration is not essential in the scalar case but will be in the vectorial setting discussed below.

We may use $\psi=d_{t} U^{j+1}$ to verify the discrete energy law for iterates of Algorithm 2.1, by Brouwer's fixed point theorem, this also implies existence of solutions to the problem.

THEOREM 2.1 Let $k, h>0$.

(i) For every $0 \leqslant m_{1} \leqslant m_{2}<\infty$,

$$
k \sum_{j=m_{1}}^{m_{2}}\left\|d_{t} U^{j+1}\right\|_{h}^{2}+\bar{G}\left(U^{m_{2}+1}\right)=\bar{G}\left(U^{m_{1}}\right),
$$

and in particular the function $m \mapsto \bar{G}\left(U^{m}\right)$ is nonincreasing. Moreover,

$$
\left\|U^{m_{2}}-U^{m_{1}}\right\|_{h}^{2} \leqslant \bar{G}\left(u_{0}\right)\left|t_{m_{2}}-t_{m_{1}}\right| .
$$

(ii) The only stationary points are constant functions.

Proof. The first part of assertion (i) follows from choosing $\psi=d_{t} U^{j+1}$ in 2.12]. The asserted inequality follows from

$$
\begin{aligned}
\left\|U^{m_{2}}-U^{m_{1}}\right\|_{h} & \leqslant k \sum_{j=m_{1}}^{m_{2}-1}\left\|d_{t} U^{j+1}\right\|_{h} \\
& \leqslant\left(k \sum_{j=m_{1}}^{m_{2}-1}\right)^{1 / 2}\left(k \sum_{j=m_{1}}^{m_{2}-1}\left\|d_{t} U^{j+1}\right\|_{h}^{2}\right)^{1 / 2} \leqslant\left|t_{m_{2}}-t_{m_{1}}\right|^{1 / 2}\left[\bar{G}\left(U^{m_{1}}\right)\right]^{1 / 2} .
\end{aligned}
$$


It remains to prove assertion (ii): by (i), we conclude $\lim _{j \rightarrow \infty}\left\|d_{t} U^{j}\right\|_{L^{2}}=0$, whence, by definition of $\tilde{\gamma}(\cdot, \cdot)$, the limit $U^{*}=\lim _{j \rightarrow \infty} U^{j} \in V_{h}$ solves

$$
\left(\left(\begin{array}{l}
g\left(\left|U_{x}^{*}\right|^{2}\right) U_{x}^{*} \\
g\left(\left|U_{y}^{*}\right|^{2}\right) U_{y}^{*}
\end{array}\right), \nabla \psi\right)=0 \quad \forall \psi \in V_{h},
$$

and thus is a critical point of $\bar{G}: V_{h} \rightarrow \mathbb{R}$. Because of Assumption 2.1.i) and $\left|\nabla U^{*}\right|<\infty$, as $h>0$, this implies the assertion. (1.1).

We refer to [26, Cor. 4.2] for a corresponding asymptotic result for a different discretization of

\subsection{Scalar Perona-Malik-numerical experiments}

Besides the energy decay property, supposed smooth solutions to 1.1 satisfy $L^{p}$-estimates, and a maximum principle [44, 57, 33]; according to [33], the behavior of gradients differs: for $M=1$, the total variation of solutions decreases, and strictly increasing behavior of $t \mapsto\|\nabla u(t)\|_{L^{\infty}}$ for supercritical initial data can be shown; for $M \geqslant 2$, (abstract) counterexamples are given which show failure of the latter two properties for gradients of the solution. We illustrate failure of a supercritical reverse maximum principle for $M=2$, which states $\frac{\mathrm{d}}{\mathrm{d} t}\|\nabla u(t, \cdot)\|_{L^{\infty}}>0$ for $\left\|\nabla u_{0}\right\|_{L^{\infty}}>1$ sufficiently large.

EXAMPle 2.1 Let $\Omega:=\left\{(x, y) \in \mathbb{R}^{2}: x^{2}+y^{2}<1\right\}, T=1$, and $u_{0}(x, y)=f(r)$, where $r=\left(x^{2}+y^{2}\right)^{1 / 2}$ and

$$
f(r)=\frac{1}{9}(2 r-1)^{9}-\frac{2}{5}(2 r-1)^{5}+(2 r-1) .
$$

The triangulations $\mathcal{T}_{h}^{(j)}$ consist of 4096 and 16384 triangles for $j=1$ and $j=2$, respectively, and define interior approximations of the domain $\Omega$. We use $h_{j}=2^{-(4+j)}$ and $k=h_{j}^{2} / 10$ for $j=1,2, \ldots, 5$. Throughout this example, we employ $\kappa=1$.

Example 2.1 has been constructed in [33, Example 3] in such a way that if there exists a radial $C^{2}$ solution of the Perona-Malik equation in $\Omega$ subject to the given initial data then there exists $\delta>0$ such that for all $t \in(0, \delta)$,

$$
\|\nabla u(t, \cdot)\|_{L^{\infty}}<\left\|\nabla u_{0}(\cdot)\right\|_{L^{\infty}}
$$

which contradicts the validity of a supercritical reverse maximum principle for the gradient. Figure 2.1 displays snapshots of the numerical approximations obtained with Algorithm 2.1 for $t=0,0.01,0.02$. The numerical solution does not seem to be radially symmetric for $t=0.01$ and $t=0.02$, and we observe a 'staircasing effect'. Nevertheless, as predicted in [33], the $W^{1, \infty}$ seminorm is decreasing at small times as can be seen in Figure 2.2, more precisely, for the discrete time derivative $d_{t}\left\|\nabla U^{1}\right\|_{L^{\infty}}$, we successively compute the values

$$
d_{t}\left\|\nabla U^{1}\right\|_{L^{\infty}}=-23.039,-22.4700,-20.0971,-18.5080,-17.9046
$$

for $h_{j}=2^{-(4+j)}, 1 \leqslant j \leqslant 5$, respectively. 

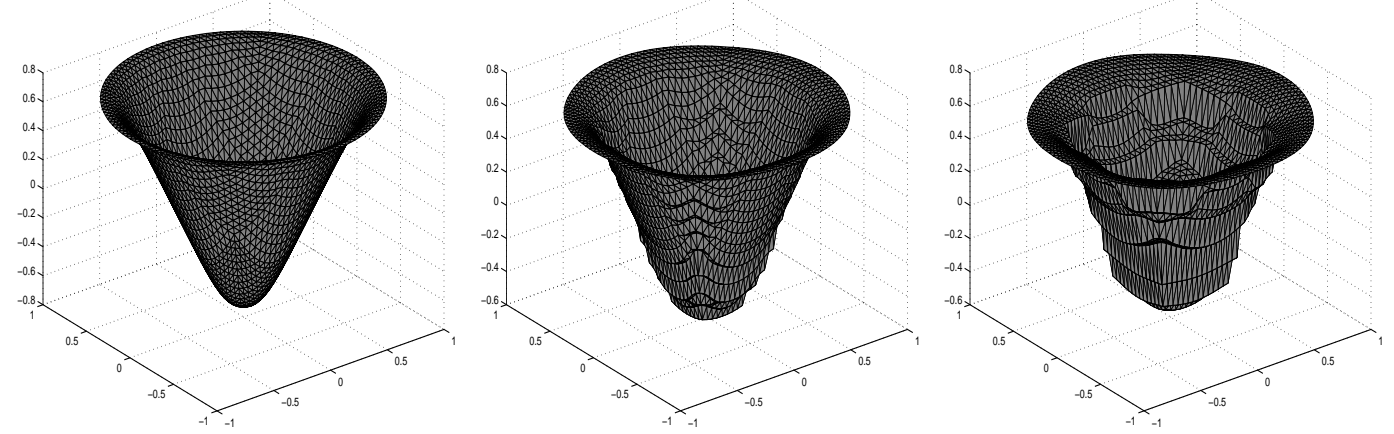

FIG. 2.1. Numerical solution in Example 2.1 for $t=0, t=0.01$, and $t=0.02$ on the triangulation $\mathcal{T}_{h}^{(1)}$.

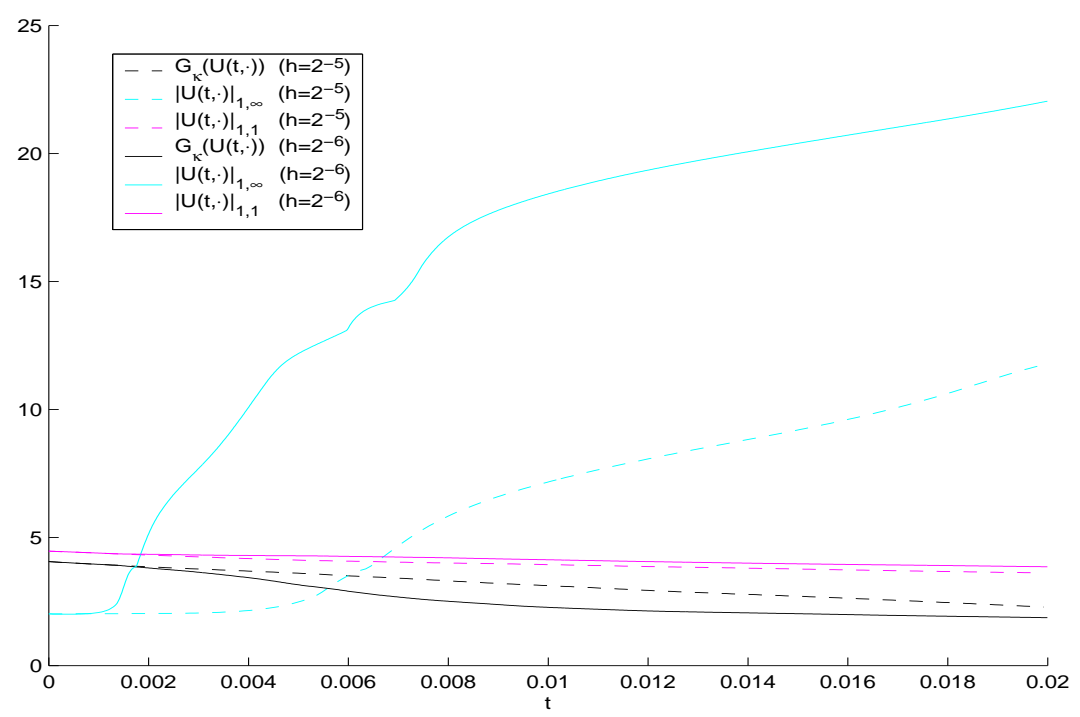

FIG. 2.2. Energy $\bar{G}\left(u_{h}(t, \cdot)\right), W^{1,1}$ seminorm $\left\|\nabla U^{j}\right\|_{1}$, and $W^{1, \infty}$ seminorm $\left\|\nabla U^{j}\right\|_{\infty}$ of the numerical approximations in Example 2.1 on the triangulations $\mathcal{T}_{h}^{(1)}\left(h=2^{-5}\right)$ and $\mathcal{T}_{h}^{(2)}\left(h=2^{-6}\right)$.

We remark that even though this example is critical in the sense that it eventually leads to large gradients, our approximation scheme guarantees a decreasing energy (cf. Figure 2.2). We also displayed the $W^{1,1}$ seminorm of the numerical approximations as functions of time and observe in the same Figure 2.2 that they are decreasing.

The following numerical experiments display advantages of the Perona-Malik evolution over (regularized) $T V$-flow, where we put $\varepsilon=h$ in 2.3). The next example compares evolution of geometric objects by Perona-Malik and $T V$-flow.

EXAMPLE 2.2 Let $\Omega=(-1 / 2,1 / 2)^{2}$ and $u_{0}(x)=\chi_{K}(x)$, where

(a) for $d=1 / 5$ and $B_{r}(a, b)=\left\{(x, y) \in \mathbb{R}^{2}:(x-a)^{2}+(y-b)^{2}<r^{2}\right\}$

$$
K=B_{1 / 8}(0,2 d / \sqrt{3}) \cup B_{1 / 8}(-d, \pm d / \sqrt{3}) ;
$$



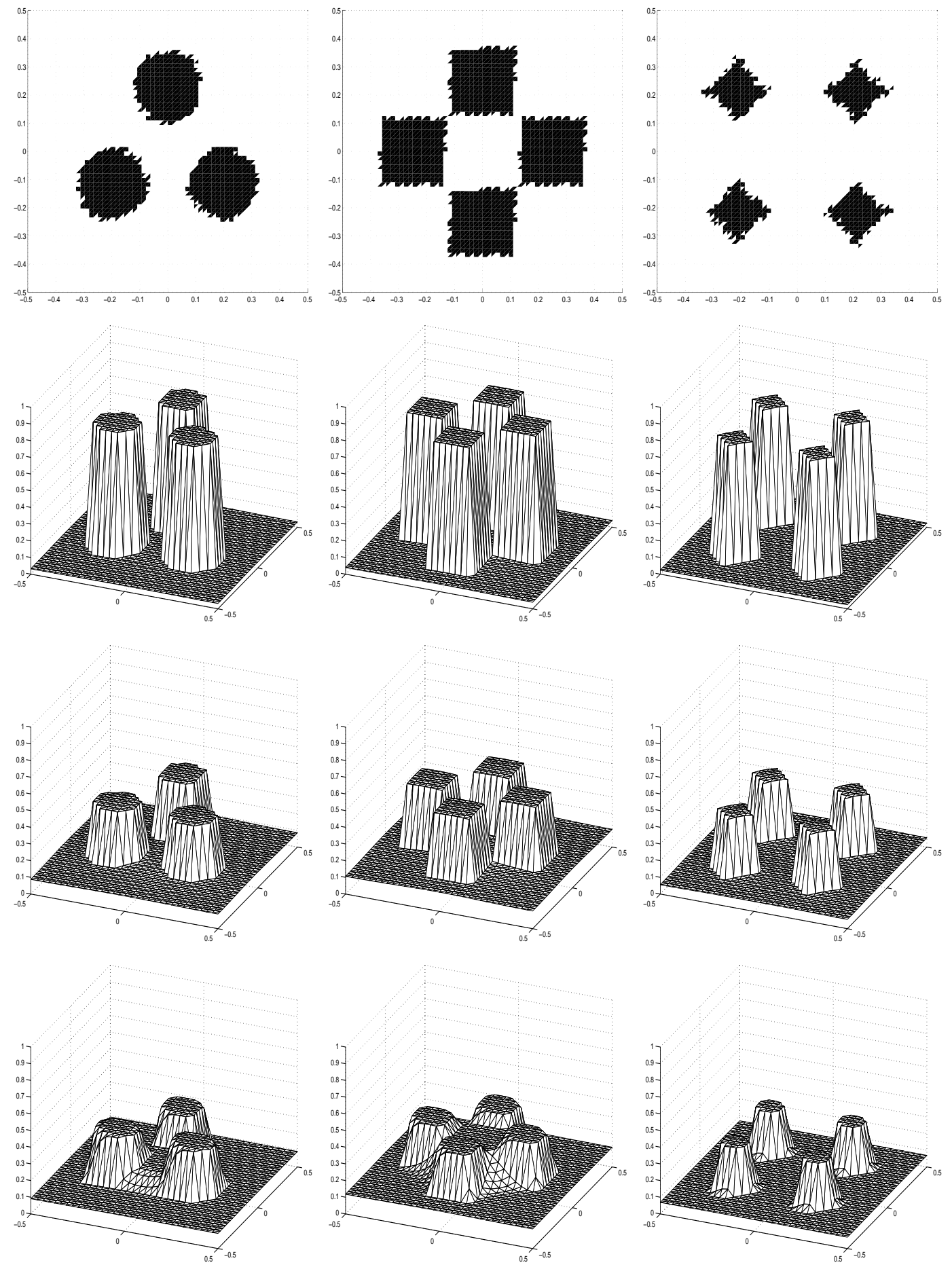
FIG. 2.3. Nodal interpolant of the initial data defined in (a)-(c) of Example 2.2 (first row). Numerical approximation of Perona-Malik equation for $t=0.15, t=0.15$, and $t=0.1$ (second row), and $t=0.3, t=0.3, t=0.175$ (third row) respectively, in (a)-(c) of Example 2.2 Developed initial data for the approximation of regularized $T V$-flow for $t=0.035$, $t=0.035, t=0.025$ in (a)-(c) of Example 2.2 for $\varepsilon=h$ (last row).

(b) for $d=1 / 4$ and $B_{r}^{\infty}(a, b)=\left\{(x, y) \in \mathbb{R}^{2}:|x-a|,|y-b|<r\right\}$

$$
K=B_{1 / 8}^{\infty}(0, \pm d) \cup B_{1 / 8}^{\infty}( \pm d, 0)
$$

(c) for $s=2 / 7$ and $r=2 / 13$

$$
K=B_{s}^{\infty}(0,0) \backslash\left(B_{r}(0,0) \cup B_{r}( \pm s, s) \cup B_{r}(-s, \pm s) \cup B_{r}(0, \pm s) \cup B_{r}( \pm s, 0)\right) .
$$

We let $\mathcal{T}_{h}$ be a triangulation of $\Omega$ consisting of halved squares along the direction $(1,1)$ and of maximal diameter $h=2^{-5}$.

We ran Algorithm 2.1 for the initial data specified in (a)-(c) of Example 2.2. The first row in Figure 2.3 displays the initial data, i.e., the nodal interpolant of the functions defined in Example 2.2 a)-(c). The second resp. third row show the numerical approximation at intermediate times $t \approx 0.15$ resp. later times $t \approx 0.3$. Diffusion of structures occurs much faster for the regularized $T V$-flow (last row), for which snapshots of the numerical solutions at time $t \approx 0.035$ for the different initial data specified are shown.

Qualitative properties of evolving sets in $\mathbb{R}^{2}$ by $T V$-flow have been studied analytically in [5. Chapter 4], and numerically in [31]. Hence, (i) linear reduction of height of characteristic functions, and (ii) change of corresponding supports built from nonconvex sets or close neighboring convex sets, as well as (iii) a rounding effect of corners are well-known properties of the $T V$ flow. In contrast, no corresponding study on qualitative properties for the Perona-Malik equation is available, where the numerical studies in rows two and three of Figure 2.3 evidence (i) significantly slower reduction of height of characteristic functions, (ii) invariance of nonconvex supports in time, and (iii) conservation of corners/edges - at least for much longer time ranges than in the case of $T V$-flow.

Our final numerical experiment for the scalar Perona-Malik equation indicates that characteristic functions remain characteristic functions in the continuous case, and 'coarsening rates' depend on the spatial discretization width $h>0$.

EXAMPLE 2.3 Set $\Omega=(-1 / 2,1 / 2)^{2}$ and $u_{0}(\mathbf{x})=\chi_{K}(\mathbf{x}), \mathbf{x} \in \Omega$, where

$$
K=B_{1 / 6}(-1 / 6,-1 / 6) \cup B_{1 / 6}(1 / 6,1 / 6) .
$$

We employ three different triangulations $\mathcal{T}_{h}^{(j)}$ of $\Omega$ which consist of 512, 2048, and 8192 triangles which are halved squares along the direction $(1,1)$ for $j=4,5,6$, respectively (cf. Figure 2.5). Moreover, we used $\kappa=1$ and $k=h_{j}^{2} / 10$, where $h_{j}=\sqrt{2} 2^{-j}$ for $j=4,5,6$.

The upper row in Figure 2.4 displays snapshots of the numerical solution on the triangulation $\mathcal{T}_{h}^{(4)}$ for $t=0, t=0.1$, and $t=0.2$. We observe that the profile of the initial $u_{0}$ is preserved for a positive time, only the height changes until $t \approx 0.3$, when a constant (stationary) state is attained. The qualitative behavior is identical for the higher resolution defined by the triangulation $\mathcal{T}_{h}^{(5)}$. 

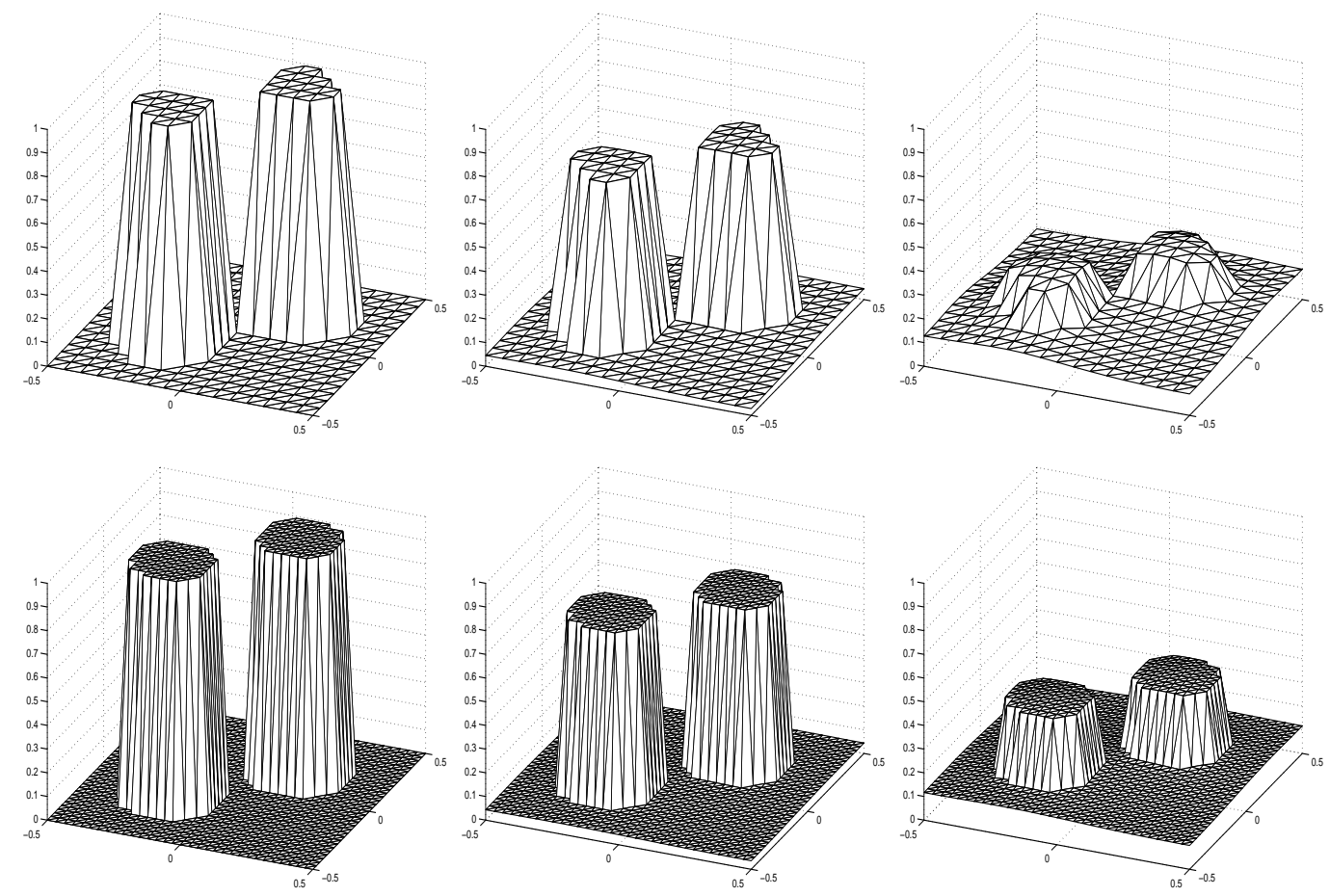

FIG. 2.4. Numerical approximation $U(t, \cdot)$ for $t=0, t=0.1$, and $t=0.2$ with $j=4$ (upper row) and for $t=0, t=0.2$, and $t=0.4$ with $j=5$ (lower row) in Example 2.3

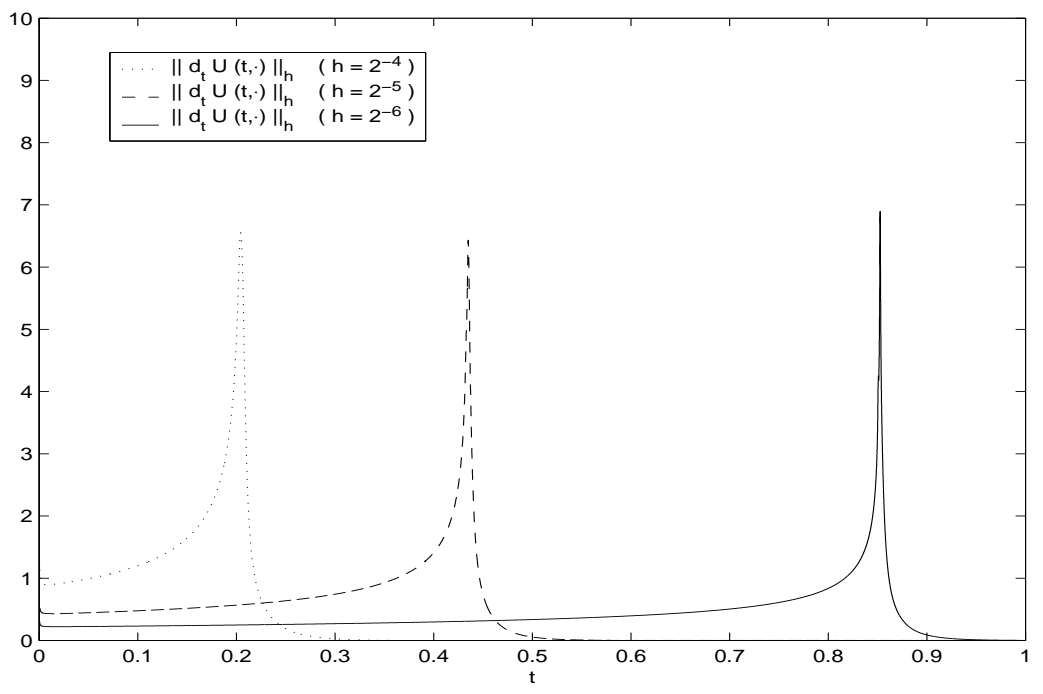

FIG. 2.5. Quantity $\left\|d_{t} U(t, \cdot)\right\|_{h}$ in Example 2.3 for the numerical approximation $U$ defined through $j=4,5,6$. 
Snapshots of the evolution are shown in the lower row of Figure 2.4 for $t=0, t=0.2$, and $t=0.4$. Again, the profile of the starting value $u_{0}$ is preserved but here for a significantly longer time (until $t \approx 0.5$ ). Thus, the time when the numerical approximation attains a constant state depends on the triangulation used. This is in good agreement with the coarsening rates derived in [29]. The behavior can also be deduced from Figure 2.5 which displays the quantity $\left\|d_{t} U(t, \cdot)\right\|_{h}$ as a function of $t \in(0,1)$ for the three triangulations $\mathcal{T}_{h}^{(j)}$ with $j=4,5,6$. Finally, we remark that for the three employed triangulations we computed the values

$$
\left(k \sum_{\ell=1}^{L}\left\|d_{t} U^{\ell}\right\|_{h}^{2}\right)^{1 / 2}=0.9311,0.7359,0.5841,
$$

which provide further indication that, as $h$ tends to zero, the initial characteristic function $u_{0}$ is a stationary state of the scalar Perona-Malik model. Note that this conjecture is no contradiction with Theorem 2.1(ii).

\section{Mappings into the sphere: one-harmonic map heat flow vs. Perona-Malik evolution}

We verify stability and an energy law for existing solutions to Algorithm 1.1. Let

$$
\hat{G}(\mathbf{u})=\sum_{\substack{1 \leqslant k \leqslant M \\ 1 \leqslant l \leqslant 3}} \int_{\Omega} \phi\left(\left|\partial_{k} u_{l}\right|^{2}\right) \mathrm{d} \mathbf{x} .
$$

Lemma 3.1 Let $k, h>0$. Suppose that $\left|\mathbf{U}^{0}(\mathbf{z})\right|=1$ for all $\mathbf{z} \in \mathcal{N}$. Then the sequence $\left\{\mathbf{U}^{m}\right\}$ from Algorithm 1.1 satisfies, for all $0 \leqslant m<\infty$,

$$
\begin{aligned}
& \text { (i) }\left|\mathbf{U}^{m}(\mathbf{z})\right|=1 \quad \forall \mathbf{z} \in \mathcal{N}, \\
& \text { (ii) } \hat{G}\left(\mathbf{U}^{m+1}\right)+k \sum_{j=0}^{m}\left\|\overline{\mathbf{U}}^{j+1 / 2} \times \mathbf{L}^{j+1}\right\|_{h}^{2}=\hat{G}\left(\mathbf{U}^{0}\right), \\
& \text { (iii) } k \sum_{j=0}^{m}\left\|d_{t} \mathbf{U}^{j+1}\right\|_{h}^{2} \leqslant \hat{G}\left(\mathbf{U}^{0}\right) .
\end{aligned}
$$

Proof. Assertion (i) follows from choosing $\psi=\varphi_{\mathbf{z}} \overline{\mathbf{U}}^{m+1 / 2}(\mathbf{z}) \in \mathbf{V}_{h}, \mathbf{z} \in \mathcal{N}$, in 1.4). In order to verify (ii), we choose $\boldsymbol{\psi}=\boldsymbol{L}^{m+1}$ in (1.4), and $\boldsymbol{\psi}=d_{t} \mathbf{U}^{m+1}$ in (1.5), and benefit from the definition of $\tilde{\gamma}$,

$$
d_{t} \sum_{k, l} \int_{\Omega} \phi\left(\left|\partial_{k} U_{l}^{m+1}\right|^{2}\right) \mathrm{d} \mathbf{x}-\left(\overline{\mathbf{U}}^{m+1 / 2} \times\left(\overline{\mathbf{U}}^{m+1 / 2} \times \boldsymbol{L}^{m+1}\right), \boldsymbol{L}^{m+1}\right)_{h}=0 .
$$

Thanks to $(\mathbf{a} \times \mathbf{b}, \mathbf{c}):=-(\mathbf{a} \times \mathbf{c}, \mathbf{b})$ for all $\mathbf{a}, \mathbf{b}, \mathbf{c} \in \mathbb{R}^{3}$, this verifies (ii). The estimate (iii) now follows easily from (i)-(ii), on putting $\psi=d_{t} \mathbf{U}^{m+1}$, and using Young's inequality.

\section{$3.1 \quad$ Fixed point method for Algorithm 1.1}

We use a simple fixed-point iteration to solve Algorithm 1.1; the subsequent method is motivated by the substitution $k d_{t} \mathbf{U}^{j+1}=2 \mathbf{W}^{j+1}-2 \mathbf{U}^{j}$, for $\mathbf{W}^{j+1}=\mathbf{U}^{j+1 / 2}$, such that (1.4)-1.5) may be 
rewritten as follows:

$$
\begin{aligned}
& \frac{2}{k}\left(\mathbf{W}^{j+1}, \boldsymbol{\psi}\right)_{h}-\left(\mathbf{W}^{j+1} \times\left(\mathbf{W}^{j+1} \times \boldsymbol{L}^{j+1}\right), \boldsymbol{\psi}\right)_{h}=\frac{2}{k}\left(\mathbf{U}^{j}, \boldsymbol{\psi}\right)_{h} \quad \forall \boldsymbol{\psi} \in \mathbf{V}_{h}, \\
& \left(\boldsymbol{L}^{j+1}, \boldsymbol{\psi}\right)_{h}=-\left(\tilde{\gamma}\left(\nabla\left\{2 \mathbf{W}^{j+1}-\mathbf{U}^{j}\right\}, \nabla \mathbf{U}^{j}\right), \nabla \boldsymbol{\psi}\right) \quad \forall \boldsymbol{\psi} \in \mathbf{V}_{h} .
\end{aligned}
$$

Given a stopping criterion $\boldsymbol{\varepsilon}=\left\{\varepsilon_{j}\right\}_{j} \geqslant 0>\boldsymbol{0}$, the fully practical linear scheme reads as follows.

Algorithm 3.1 1. Set $\tilde{\mathbf{U}}^{0}:=\mathbf{U}^{0}$ and $\mathbf{W}^{1,0}:=\tilde{\mathbf{U}}^{0}$. Set $j:=0$ and $\ell:=0$.

2. Compute $\mathbf{W}^{j+1, \ell+1} \in \mathbf{V}_{h}$ such that for all $\boldsymbol{\psi} \in \mathbf{V}_{h}$,

$$
\begin{aligned}
& \frac{2}{k}\left(\mathbf{W}^{j+1, \ell+1}, \boldsymbol{\psi}\right)_{h}-\left(\mathbf{W}^{j+1, \ell+1} \times\left(\mathbf{W}^{j+1, \ell} \times \boldsymbol{L}^{j+1, \ell}\right), \boldsymbol{\psi}\right)_{h}=\frac{2}{k}\left(\tilde{\mathbf{U}}^{j}, \boldsymbol{\psi}\right)_{h} \quad \forall \boldsymbol{\psi} \in \mathbf{V}_{h}, \\
& \left(\boldsymbol{L}^{j+1, \ell}, \boldsymbol{\psi}\right)_{h}=-\left(\tilde{\boldsymbol{\gamma}}\left(\nabla\left\{2 \mathbf{W}^{j+1, \ell}-\tilde{\mathbf{U}}^{j}\right\}, \nabla \tilde{\mathbf{U}}^{j}\right), \nabla \boldsymbol{\psi}\right) \quad \forall \boldsymbol{\psi} \in \mathbf{V}_{h} .
\end{aligned}
$$

3. Given $\varepsilon_{j}>0$, stop if

$$
\left\|\mathbf{W}^{j+1, \ell+1}-\mathbf{W}^{j+1, \ell}\right\|_{L^{2}} \leqslant \varepsilon_{j},
$$

and set $\tilde{\mathbf{U}}^{j+1}:=2 \mathbf{W}^{j+1, \ell+1}-\tilde{\mathbf{U}}^{j} \in \mathbf{V}_{h}$.

4. Set $\ell:=\ell+1$ and go to Step 2 .

Unconditional unique solvability of 3.3 for $\mathbf{W}^{j+1, \ell+1} \in \mathbf{L}^{2}$ follows from the Lax-Milgram theorem.

Theorem 3.1 Let $\hat{G}\left(\tilde{\mathbf{U}}^{j}\right) \leqslant C$ and $\left|\tilde{\mathbf{U}}^{j}(\mathbf{z})\right|=1$ for all $\mathbf{z} \in \mathcal{N}$ and $j \geqslant 0$ in Algorithm 3.1. For all $\ell \geqslant 0$, there exists a unique solution $\mathbf{W}^{j+1, \ell+1} \in \mathbf{L}^{2}$ to $3.3-3.4$ such that $\left\|\mathbf{W}^{j+1, \ell+1}\right\|_{L^{\infty}} \leqslant 1$. Moreover there exists $C>0$ such that

$$
\left\|\boldsymbol{L}^{j+1, \ell}\right\|_{L^{\infty}} \leqslant C h^{-1} .
$$

Additionally, there exists $0 \leqslant \tilde{C}<1$ such that for $k \leqslant \tilde{C} h^{2}$, there exists $0<\Gamma<1$ with

$$
\left\|\mathbf{W}^{j+1, \ell+1}-\mathbf{W}^{j+1, \ell}\right\|_{L^{2}} \leqslant \Gamma\left\|\mathbf{W}^{j+1, \ell}-\mathbf{W}^{j+1, \ell-1}\right\|_{L^{2}}, \quad \ell \geqslant 1,
$$

and $\left|\tilde{\mathbf{U}}^{j+1}(\mathbf{z})\right|=1$ for all $\mathbf{z} \in \mathcal{N}$.

Proof. Control of $\left\|\mathbf{W}^{j+1, \ell+1}\right\|_{L^{\infty}}$ by 1 follows from 3.3$)$ on choosing $\boldsymbol{\psi}=\mathbf{W}^{j+1, \ell+1}(\mathbf{z}) \varphi_{\mathbf{z}}$.

To verify $\left\|\boldsymbol{L}^{j+1, \ell}\right\|_{L^{\infty}} \leqslant C h^{-1}$, choose $\mathbf{z} \in \mathcal{N}$ such that $\left\|\boldsymbol{L}^{j+1, \ell}\right\|_{L^{\infty}}=\left|\boldsymbol{L}^{j+1, \ell}(\mathbf{z})\right|$. Upon choosing $\boldsymbol{\psi}=\boldsymbol{L}^{j+1, \ell}(\mathbf{z}) \varphi_{\mathbf{z}}$ in 3.2 , we have

$$
\begin{aligned}
\beta_{\mathbf{z}}\left|\boldsymbol{L}^{j+1, \ell}(\mathbf{z})\right|^{2} & \leqslant\left|\left(\tilde{\boldsymbol{\gamma}}\left(\nabla\left\{2 \mathbf{W}^{j+1}-\tilde{\mathbf{U}}^{j}\right\}, \nabla \tilde{\mathbf{U}}^{j}\right), \nabla \varphi_{\mathbf{z}}\right)\right|\left|\boldsymbol{L}^{j+1, \ell}(\mathbf{z})\right| \\
& \leqslant\left|\boldsymbol{L}^{j+1, \ell}(\mathbf{z})\right| \sum_{K \in \mathcal{T}_{h}, \mathbf{z} \in \bar{K}}|K|\left|\nabla \varphi_{\mathbf{z}}\right|\left|\tilde{\boldsymbol{\gamma}}\left(\nabla\left\{2 \mathbf{W}^{j+1}-\tilde{\mathbf{U}}^{j}\right\}, \nabla \tilde{\mathbf{U}}^{j}\right)\right|,
\end{aligned}
$$

with $c h^{M} \leqslant \beta_{\mathbf{z}},|K| \leqslant C h^{M}$. Since the sum is finite and $\tilde{\boldsymbol{\gamma}} \leqslant C$, this implies $\left|\boldsymbol{L}^{j+1, \ell}(\mathbf{z})\right| \leqslant C h^{-1}$.

Subtraction of two subsequent equations in the fixed-point iteration yields, for every $\ell \geqslant 1$ and all $\psi \in \mathbf{V}_{h}$,

$$
\begin{aligned}
\frac{1}{k}\left(\mathbf{E}_{j+1}^{\ell+1}, \boldsymbol{\psi}\right)_{h}+\left(\mathbf{E}_{j+1}^{\ell+1} \times\left(\mathbf{W}^{j+1, \ell} \times \boldsymbol{L}^{j+1, \ell}\right), \boldsymbol{\psi}\right)_{h}+\left(\mathbf{W}^{j+1, \ell} \times\left(\mathbf{E}_{j+1}^{\ell} \times \boldsymbol{L}^{j+1, \ell}\right), \boldsymbol{\psi}\right)_{h} \\
+\left(\mathbf{W}^{j+1, \ell} \times\left(\mathbf{W}^{j+1, \ell} \times\left[\boldsymbol{L}^{j+1, \ell}-\boldsymbol{L}^{j+1, \ell-1}\right]\right), \boldsymbol{\psi}\right)_{h}=0 .
\end{aligned}
$$


Choosing $\psi=\mathbf{E}_{j+1}^{\ell+1}$ leads to

$$
\left\|\mathbf{E}_{j+1}^{\ell+1}\right\|_{h} \leqslant k\left\|\mathbf{W}^{j+1, \ell}\right\|_{L^{\infty}}\left\|\boldsymbol{L}^{j+1, \ell}\right\|_{L^{\infty}}\left\|\mathbf{E}_{j+1}^{\ell}\right\|_{h}+k\left\|\mathbf{W}^{j+1, \ell}\right\|_{L^{\infty}}^{2}\left\|\boldsymbol{L}^{j+1, \ell}-\boldsymbol{L}^{j+1, \ell-1}\right\|_{h} .
$$

Thanks to 3.2, the mean value theorem together with uniform boundedness of $\left|D^{2} \phi\left(|\cdot|^{2}\right)\right|$ which implies uniform Lipschitz continuity of $\tilde{\gamma}(\cdot, X)$ when $X$ is fixed, and an inverse inequality, the last term in 3.8 may be bounded by

$$
C k h^{-1}\left\|\nabla \mathbf{E}_{j+1}^{\ell}\right\|_{L^{2}} \leqslant C k h^{-2}\left\|\mathbf{E}_{j+1}^{\ell}\right\|_{L^{2}} .
$$

A combination of the estimates then yields assertion 3.7 . Finally, if $\tilde{\mathbf{U}}^{j+1}=2 \mathbf{W}^{j+1, \ell+1}-\tilde{\mathbf{U}}^{j}$, equation (3.3) can be written as

$$
\left(d_{t} \tilde{\mathbf{U}}^{j+1}, \boldsymbol{\psi}\right)_{h}-\left(\overline{\tilde{\mathbf{U}}^{j+1 / 2}} \times\left(\overline{\tilde{\mathbf{U}}}^{j+1 / 2} \times \boldsymbol{L}^{j+1, \ell}\right), \boldsymbol{\psi}\right)_{h}=0 .
$$

The choice $\psi=\overline{\tilde{\mathbf{U}}}^{j+1 / 2}(\mathbf{z}) \varphi_{\mathbf{z}}$ then implies $d_{t}\left|\tilde{\mathbf{U}}^{j+1}(\mathbf{z})\right|^{2}=0$.

\subsection{Computational experiments}

Numerical experiments with Algorithm 1.1 show that evolved edges/structures are preserved for a long time.

EXAMPLE 3.1 (a) Let $\Omega:=(-1,1)^{2}, T=1 / 2$, and $\mathbf{u}_{0}$ be as in the left upper plot in Figure 3.1 . We choose a triangulation $\mathcal{T}_{h}$ consisting of 2048 triangles which are halved squares (along the direction $(1,1))$ and with 1089 nodes. Hence, $h=\sqrt{2} 2^{-4}$ and we set $k=h^{2} / 10$.

(b) Let $\Omega, T$, and $\mathcal{T}_{h}$ be as in (a) and let $\mathbf{u}_{0}$ be the perturbation of the initial data of (a) shown in the left plot of Figure 3.4

Figure 3.1 shows the evolution defined by the Perona-Malik equation into the sphere in Example 3.1 a) and computed with Algorithm 1.1. We observe that the sharp interfaces separating regions in which $\mathbf{u}=(1,0,0)$ from those in which $\mathbf{u}=(0,1,0)$ are preserved for a long time $t=0.44688$ before all vectors start to align. This is different if regularized one-harmonic flow $(\varepsilon=h)$ is applied to the initial data of Example 3.1 (a) as one can deduce from the snapshots of the numerical solutions for different times shown in Figure 3.2 for its calculation, we used a consistent, stable scheme constructed in [9], where iterates satisfy an analogue of Lemma 3.1. The vectors immediately start to align, and at $t=0.15469$ no sharp interfaces are observable. In Figure 3.3 (left) we displayed the first component of the vectors $\mathbf{u}(t, \mathbf{x})$ for $\mathbf{x} \in\{(-1 / 4,0),(1 / 2,0)\}$ as functions of $t>0$. This allows for a quantitative comparison of the speed of the alignment of the vectors displayed in Figures 3.1 and 3.2 We observe an approximately linear change in the direction for $0 \leqslant t \leqslant 2$ in the case of (regularized) one-harmonic map heat flow into the unit sphere, while the rotation is slowed down for Perona-Malik evolution on the sphere. The perturbed initial data shown in the left plot of Figure 3.4 is denoised at $t=0.020313$ but (practical) discontinuities are preserved.

We conclude our discussion on the qualitative behavior of the vectorial Perona-Malik flow into spheres with an example that shows that a 'staircasing effect' occurs as in the scalar case. The next example evolves smooth initial data, leading to iterates with large gradients. 

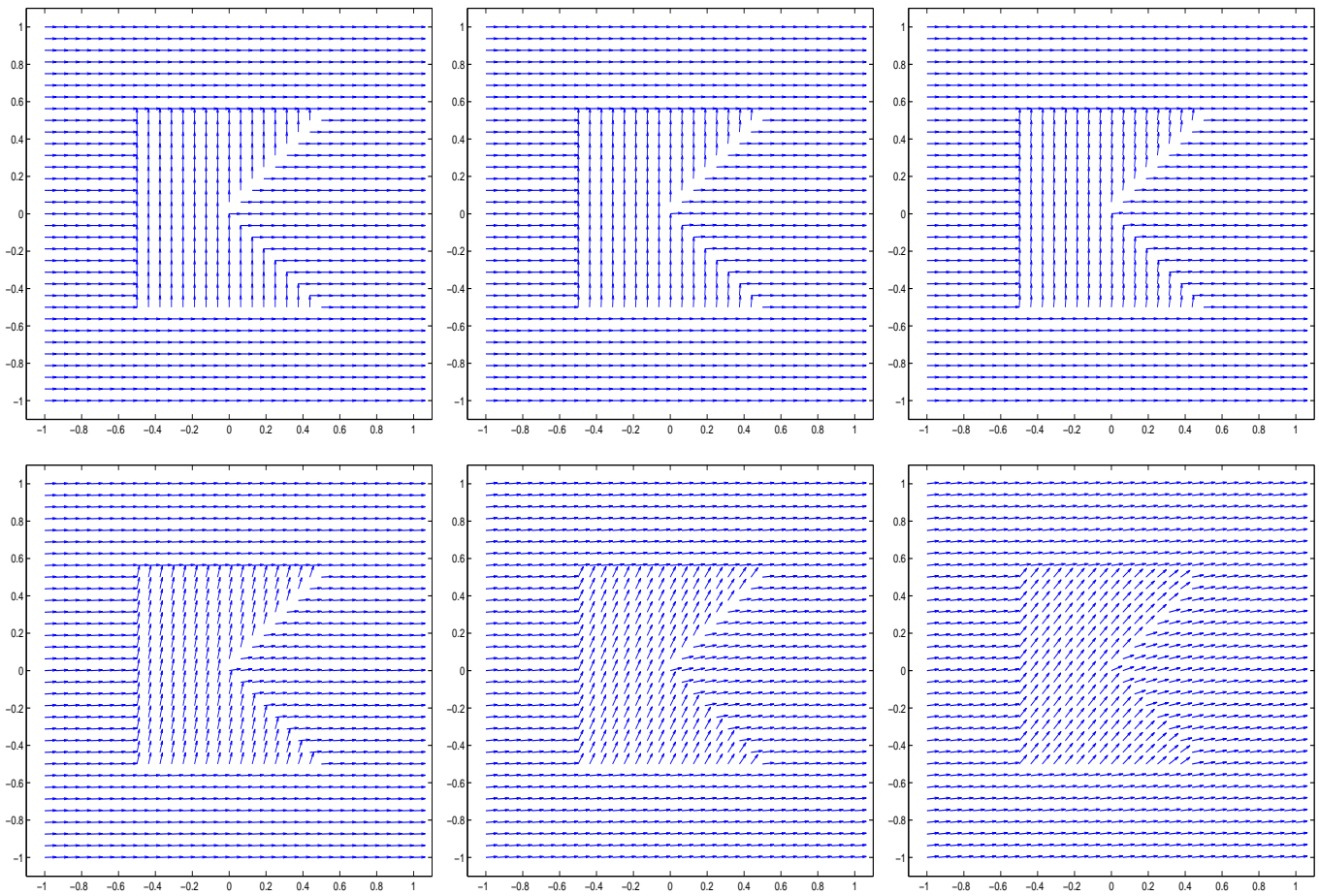

FIG. 3.1. First two components of the numerical solutions $\mathbf{u}(t, \cdot)$ for $t=0,0.085938,0.15469,0.44688,0.77344,0.99688$ in Example 3.1 a) obtained with Perona-Malik evolution on the sphere.
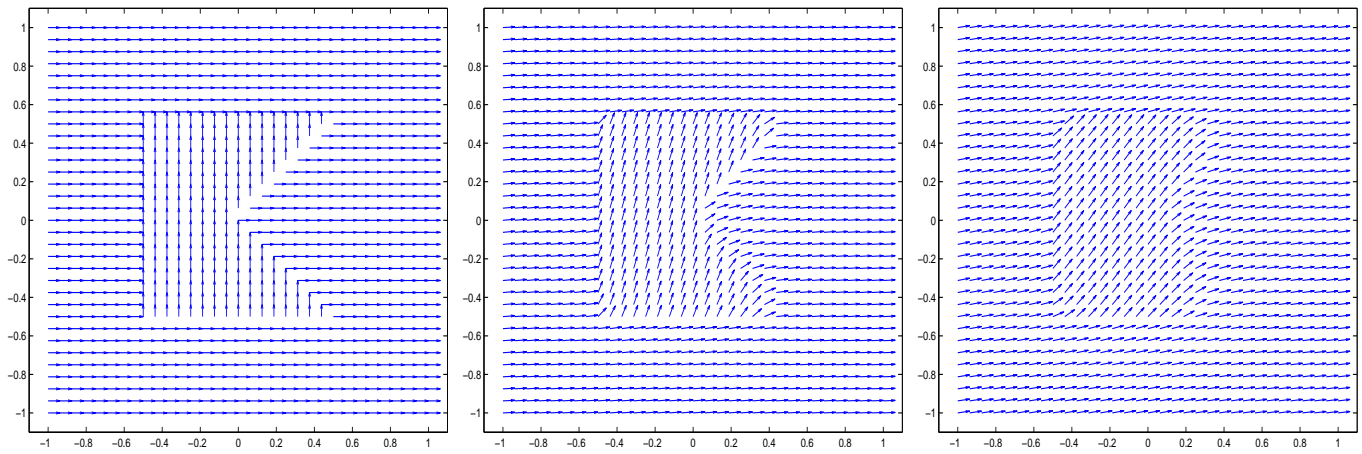

FIG. 3.2. First two components of the numerical solution $\mathbf{u}(t, \cdot)$ for $t=0,0.085938,0.15469$ in Example 3.1 a) obtained with regularized one-harmonic map heat flow $(\varepsilon=h)$.

EXAmple 3.2 Let $\Omega=(-1,1)^{2}$ and $\mathbf{u}_{0}(\mathbf{x})=(\cos \pi|\mathbf{x}|, \sin \pi|\mathbf{x}|, 0)$. Three uniform triangulations $\mathcal{T}_{h}^{(j)}$ for $j=4,5,6$ of $\Omega$ with maximal mesh-size $h_{j}=2 \sqrt{2} 2^{-j}$ are employed. For each $j=4,5,6$ we set $\kappa=1$ and $k=h_{j}^{2} / 10$. 

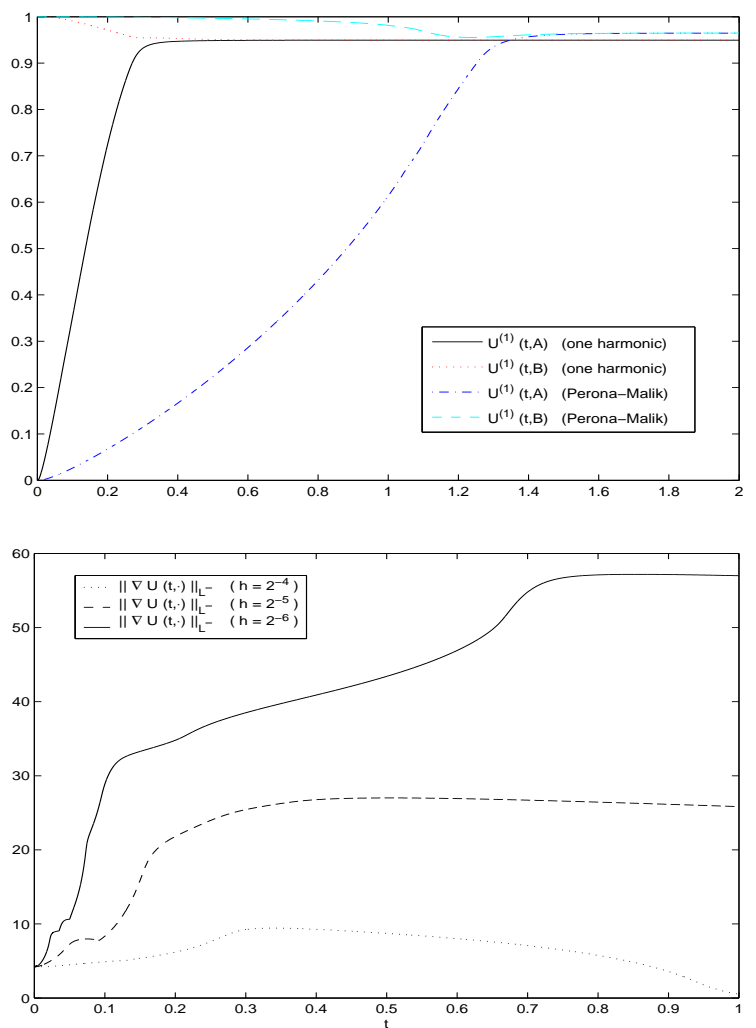

FIG. 3.3. First components $\mathbf{u}^{(1)}(t, \mathbf{A})$ and $\mathbf{u}^{(1)}(t, \mathbf{B})$ of the numerical solutions in Example 3.1 a) obtained with regularized one-harmonic map heat flow and regularized one-harmonic map heat flow into the sphere for $\mathbf{A}=(-1 / 4,0)$ and $\mathbf{B}=$ $(1 / 2,0)$ for $\varepsilon=h$ (top). Quantity $\|\nabla \mathbf{U}(t, \cdot)\|_{L^{\infty}}$ in Example 3.2 for the numerical approximation $\mathbf{U}$ defined through the triangulations $\mathcal{T}_{h}^{(j)}$ with $j=4,5,6$ (bottom).
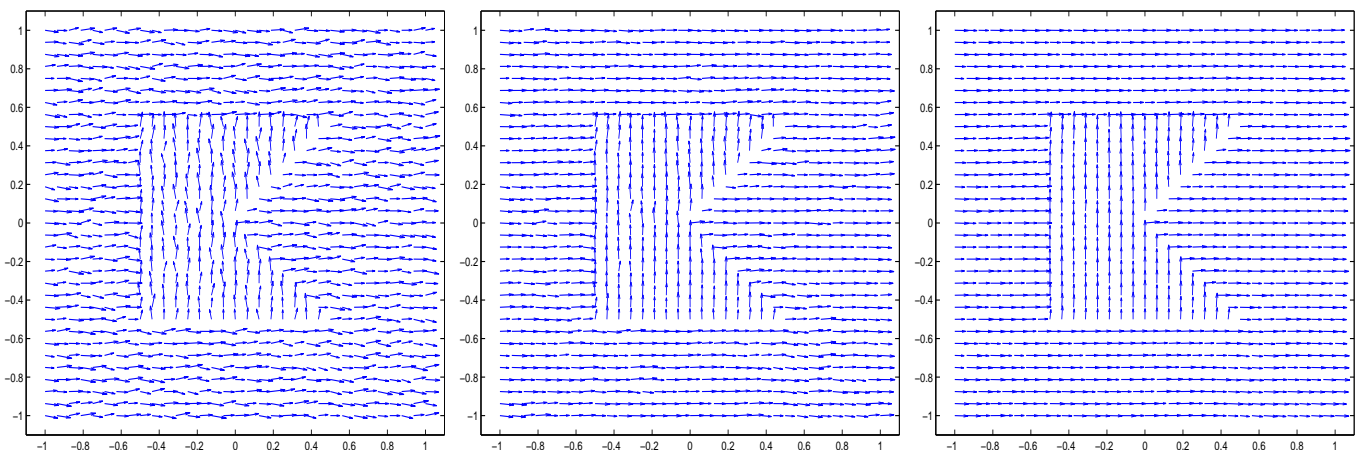

FIG. 3.4. First two components of the numerical solution $\mathbf{u}(t, \cdot)$ for $t=0,0.010156,0.020313$ in Example 3.1 b) obtained with Perona-Malik evolution on the sphere. 

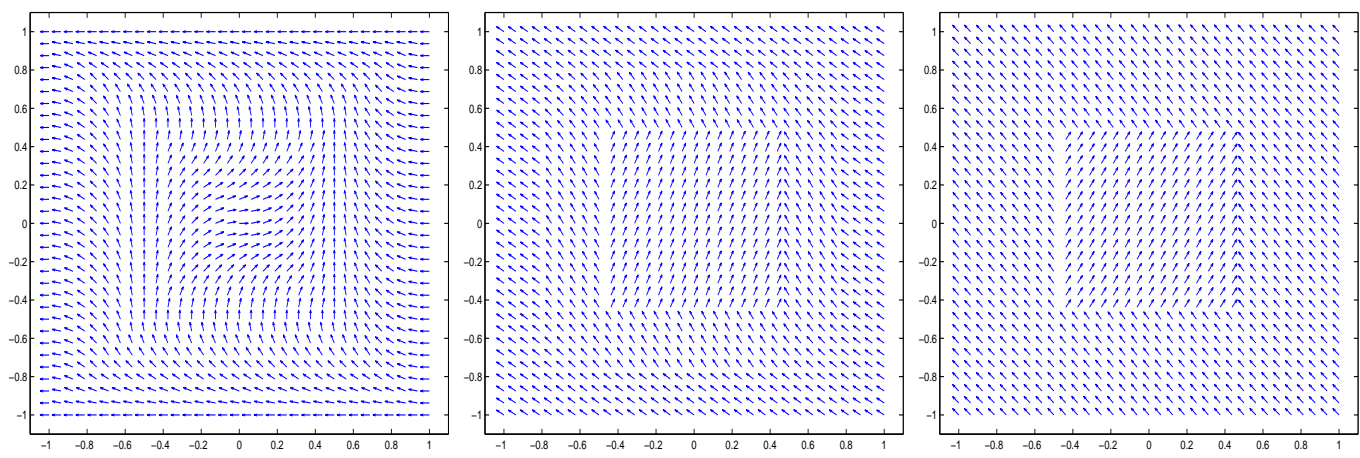

FIG. 3.5. Numerical solution $\mathbf{U}(t, \cdot)$ for $t=0, t=0.1$, and $t=0.2$ on triangulation $\mathcal{T}_{h}^{(5)}$ in Example 3.2

Figure 3.5 shows the numerical solution $\mathbf{U}(t, \cdot)$ on the triangulation $\mathcal{T}_{h}^{(5)}$ for $t=0, t=0.2$, and $t=0.38$. We see that the initially smooth vector field $\mathbf{U}^{0}$ changes during the evolution and at $t=0.38$, a piecewise constant state with a sharp interface, that separates regions in which the approximation is constant, is attained. The interface becomes sharper for finer triangulations, which is reflected in Figure 3.3 (bottom), where we plotted for $j=4,5,6$ the quantity $\|\nabla \mathbf{U}(t, \cdot)\|_{L^{\infty}}$ as function of $t$; large (almost maximal) gradients are attained for each triangulation.

\section{REFERENCES}

1. ACAR, R., \& Vogel, C. R. Analysis of bounded penalty methods for ill-posed problems. Inverse Problems 10 (1994), 1217-1229. Zbl 0809.35151 MR 1306801

2. Alvarez, L., Guichard, F., Lions, P.-L., \& Morel, J.-M. Axioms and fundamental equations of image processing. Arch. Ration. Mech. Anal. 123 (1993), 199-257. Zbl 0788.68153 MR 1225209

3. Amann, H. Time-delayed Perona-Malik type problems. Acta Math. Univ. Comen. 76 (2007), 15-38. Zbl pre05136268 MR 2331050

4. Ambrosio, L., Fusco, N., \& Pallara, D. Functions of Bounded Variation and Free Discontinuity Problems. Oxford Univ. Press, New York (2000). Zbl 0957.49001 MR 1857292

5. Andreu-Vaillo, F., Caselles, V., \& Mazón, J. M. Parabolic Quasilinear Equations Minimizing Linear Growth Functionals. Birkhäuser (2004). Zbl 1053.35002 MR 2033382

6. Barrett, J. W., Bartels, S., Feng, X., \& Prohl, A. A convergent and constraint-preserving finite element method for the $p$-harmonic flow into spheres. SIAM J. Numer. Anal. 45 (2007), 905-927. MR 2318794

7. BARTELS, S. Stability and convergence of finite element approximation schemes for harmonic maps. SIAM J. Numer. Anal. 43 (2005), 220-238. Zbl 1090.35014 MR 2177142

8. BARtels, S., \& Prohl, A. Constraint preserving implicit finite element discretization of harmonic map flow into spheres. Math. Comp. 16 (2007), 1847-1859. Zbl pre05190892

9. Bartels, S., \& Prohl, A. Convergence of an implicit, constraint preserving finite element discretization of $p$-harmonic heat flow into sphere. Manuscript (2006).

10. Belahmidi, A., \& Chambolle, A. Time-delay regularization of anisotropic diffusion and image processing. M2AN 39 (2005), 231-251. Zbl 1101.68102 MR 2143948 
11. Bellettini, G., \& Fusco, G. A regularized Perona-Malik functional: some aspects of the gradient dynamics. Equadiff 2003 (Hasselt, 2003), F. Dumortier, H. Broer et al. (eds.), World Sci. (2003), 639-644. Zbl 1108.35078 MR 2185104

12. Bellettini, G., \& Fusco, G. The $\Gamma$-limit and the related gradient flow for singular perturbation functionals of Perona-Malik type. http://cvgmt.sns.it/papers/belfusa (2004).

13. Bellettini, G., Fusco, G., \& Guglielmi, N. A concept of solution and numerical experiments for forward-backward diffusion equations. Discr. Contin. Dyn. Syst. 16 (2006), 783-842. Zbl 1105.35007 MR 2257160

14. Bellettini, G., Novaga, M., \& Paolini, E. Global solutions to the gradient flow equation of a nonconvex functional. SIAM J. Math. Anal. 37 (2006), 1657-1687. Zbl 1109.35050 MR 2215602

15. Bellettini, G., Novaga, M., Paolini, M., \& Tornese, C. Convergence of discrete schemes for the Perona-Malik equation. http://cvgmt.sns.it/papers/belnovpao06 (2006).

16. Bertozzi, A. L., \& Greer, J. B. Low-curvature image simplifiers: global regularity of smooth solutions and Laplacian limiting schemes. Comm. Pure Appl. Math. 57 (2004), 764-790. Zbl 1058.35083 MR 2038116

17. Bouchitté, G., Braides, A., \& Buttazzo, G. Regular approximation of free-discontinuity problems. Math. Models Methods Appl. Sci. 10 (2000), 1073-1097. Zbl 1009.49012 MR 1780150

\begin{tabular}{l} 
18. BRaides, A. Approximation of Free-Discontinuity Problems. Springer (1998). Zbl 0909.49001 \\
\hline
\end{tabular} MR 1651773

19. CAtté, F., Lions, P.-L., Morel, J.-M., \& Coll, T. Image selective smoothing and edge detection by nonlinear diffusion. SIAM J. Numer. Anal. 29 (1992), 182-193. Zbl 0746.65091 MR 1149092

20. Chambolle, A. An algorithm for total variation minimization and applications. J. Math. Imag. Vision 20 (2004), 49-97. MR 2049783

21. Chambolle, A., \& LiOnS, P.-L. Image recovery via total variation minimization and related problems. Numer. Math. 76 (1997), 167-188. Zbl 0874.68299 MR 1440119

22. ChAn, T., \& SHEN, J. Variational restoration of nonflat image features: models and algorithms. SIAM J. Appl. Math. 61 (2000), 1338-1361. Zbl 1011.94001 MR 1813683

23. Chen, Y., Hong, M.-C., \& HungerbüHLER, N. Heat flow of $p$-harmonic maps with values into spheres. Math. Z. 215 (1994), 25-35. Zbl 0793.53049 MR 1254812

24. Demoulini, S. Young measure solutions for a nonlinear parabolic equation of forward-backward type. SIAM J. Math. Anal. 27 (1996), 376-403. Zbl 0851.35066 MR 1377480

25. Dobson, D. C., \& Vogel, C. R. Convergence of an iterative method for total variation denoising. SIAM J. Numer. Anal. 34 (1997), 1779-1791. Zbl 0898.65034 MR 1472196

26. Elliott, C. M., Gawron, B., Maier-PaApe, S., \& VAn Vleck, E. S. Discrete dynamics for convex and non-convex smoothing functionals in PDE based image restoration. Comm. Pure Appl. Anal. 5 (2006), 181-200. MR 2190784

27. Esedoglu, S. An analysis of the Perona-Malik scheme. Comm. Pure Appl. Math. 54 (2001), 14421487. Zbl 1031.68133 MR 1852979

28. Esedoglu, S. Stability properties of Perona-Malik scheme. SIAM J. Numer. Anal. 44 (2006), 12971313. Zbl 05167775 MR 2231865

29. Esedoglu, S., \& Greer, J. Upper bounds on the coarsening rate of discrete, ill-posed nonlinear diffusion equations. http://math.nyu.edu/greer (2006).

30. Fierro, F., Goglione, R., \& PAolini, M. Numerical simulations of mean curvature flow in presence of a nonconvex anisotropy. Math. Models Methods Appl. Sci. 4 (1998), 573-601. Zbl 0946.58014 MR 1634826

31. Feng, X., Von OehSEn, M., \& Prohl, A. Rate of convergence of regularization procedures and finite element approximations for the total variation flow. Numer. Math. 100 (2005), 441-456. Zbl 1075.65114 MR 2194526 
32. FEng, X., \& Prohl, A. Analysis of total variation flow and its finite element approximations. M2AN 37 (2003), 533-556. Zbl 1050.35004 MR 1994316

33. Ghisi, M., \& Gobbino, M. Gradient estimates for the Perona-Malik equation. Math. Ann. 337 (2007), 557-590. Zbl 05145026 MR 2274543

34. Ghisi, M., \& GobBino, M. A class of local classical solutions for the one-dimensional Perona-Malik equation. http://cvgmt.sns.it/papers/ghigob06 (2006).

35. Giaquinta, M., \& MuCCI, D. The $B V$-energy of maps into a manifold: relaxation and density results. Ann. Scuola Norm. Sup. Pisa Cl. Sci. 5 (2006), 483-548. Zbl 05151088 MR 2297721

36. Giga, Y., Kashima, Y., \& YAmaZAKi, N. Local solvability of a constrained gradient system of total variation. Abstr. Appl. Anal. 8 (2004), 651-682. Zbl 1068.35054 MR 2096945

37. Gobbino, M. Gradient flow for the one-dimensional Mumford-Shah functional. Ann. Scuola Norm. Sup. Pisa Cl. Sci. 27 (1998), 145-193. Zbl 0931.49010 MR 1658873

38. Gobbino, M. Entire solutions of the one-dimensional Perona-Malik equation. Comm. Partial Differential Equations 32 (2007), 719-743. MR 2334830

39. HANdlovicová, A., \& KRIVÁ, Z. Error estimates for finite volume scheme for Perona-Malik equation. Acta Math. Univ. Comen. 74 (2005), 79-94. Zbl 1108.35083 MR 2154399

40. Hardt, R., \& ZHOU, X. An evolution problem for linear growth functionals. Comm. Partial Differential Equations 19 (1994), 1879-1907. Zbl 0811.35061 MR 1301176

41. HungerbüHLER, N. Heat flow into spheres for a class of energies. Variational Problems in Riemannian Geometry. Progr. Nonlinear Differential Eqnuations Appl. 59, Birkhäuser (2004), 45-65. Z Zbl 1063.58010 MR 2076266

42. KaCUR, J., \& Mikula, K. Solution of nonlinear diffusion appearing in image processing and edge detection. Appl. Numer. Math. 17 (1995), 47-59. Zbl 0823.65089|| MR 1335517

43. Kawohl, B. From Mumford-Shah to Perona-Malik in image processing. Math. Methods Appl. Sci. 27 (2004), 1803-1814. Zbl 1060.35054 MR 2087298

44. KAwohl, B., \& KuteV, N. Maximum and comparison principle for one-dimensional anisotropic diffusion. Math. Ann. 311 (1998), 107-123. Zbl 0909.35025 MR 1624275

45. Kichenassamy, S. The Perona-Malik paradox. SIAM J. Appl. Math. 57 (1997), 1328-1342. Zbl 0887.35071 MR 1470926

46. Mester, M. Mathematische Methoden zur Bildverarbeitung. Ph.D. thesis, Univ. Köln (2004).

47. Mikula, K., \& Ramarosy, N. Semi-implicit finite volume scheme for solving nonlinear diffusion equations in image processing. Numer. Math. 89 (2001), 561-590. Zbl 1013.65094 MR 1864431

48. Morini, M. Sequences of singularly perturbed functionals generating free-discontinuity problems. SIAM J. Math. Anal. 35 (2003), 759-805. Zbl 1058.49021 MR 2048406

49. Nitzberg, M., \& Shiota, T. Nonlinear image filtering with edge and corner enhancement. IEEE Trans. Pattern Anal. Mach. Intell. 14 (1992), 826-833.

50. Perona, P., \& MALIK, J. Scale-space and edge detection using anisotropic diffusion. IEEE Trans. Pattern Anal. Mach. Intell. 12 (1992), 629-639.

51. Rudin, L., Osher, S., \& FAtemi, E. Nonlinear total variation based noise removal algorithms. Phys. D 60 (1992), 259-268. Zbl 0780.49028

52. Struwe, M. Geometric evolution problems. Nonlinear Partial Differential Equations in Differential Geometry (Park City, UT, 1992), IAS/Park City Math. Ser. 2, Amer. Math. Soc. (1996), 259-339. Zbl 0847.58012 MR 1369591

53. TAheri, S., TANG, Q., \& Zhang, K. Young measure solutions and instability of the one-dimensional Perona-Malik equation. J. Math. Anal. Appl. 308 (2005), 467-490. Zbl 1081.35056 MR 2150102

54. TANG, B., SAPIRo, G., \& CASElles, V. Color image enhancement via chromaticity diffusion. IEEE Trans. Image Process. 10 (2001), 701-707. Zbl 1037.68792 
55. TSCHUMPERLÉ, D. PDE's based regularization of multivalued images and applications. Doctoral thesis, Univ. de Nice, ftp://ftp-sop.inria.fr/odyssee/Publications/PhDs/tschumperle:02.pdf (2002).

56. Vese, L. A., \& Osher, S. J. Numerical methods for $p$-harmonic flows and applications to image processing. SIAM J. Numer. Anal. 40 (2002), 2085-2104. Zbl 1035.65065 MR 1974176

57. Weickert, J. Anisotropic Diffusion in Image Processing. Teubner, Stuttgart (1998). Zbl 0886.68131 MR 1666943 